\title{
High-Throughput Identification of Organic Compounds from Polygoni Multiflori Radix Praeparata (Zhiheshouwu) by UHPLC-Q-Exactive Orbitrap-MS
}

\author{
Shaoyun Wang, Xiaozhu Sun, Shuo An, Fang Sang, Yunli Zhao * and Zhiguo Yu *D \\ School of Pharmacy, Shenyang Pharmaceutical University, Shenyang 110016, China; \\ wsy17862968828@163.com (S.W.); sxz13591173561@163.com (X.S.); a13831181394@163.com (S.A.); \\ sangfang03@163.com (F.S.) \\ * Correspondence: ylzhao@syphu.edu.cn (Y.Z.); yuzhiguo@syphu.edu.cn (Z.Y.)
}

check for updates

Citation: Wang, S.; Sun, X.; An, S.; Sang, F.; Zhao, Y.; Yu, Z.

High-Throughput Identification of

Organic Compounds from Polygoni Multiflori Radix Praeparata (Zhiheshouwu) by UHPLC-Q-Exactive Orbitrap-MS. Molecules 2021, 26, 3977. https://doi.org/10.3390/

molecules26133977

Academic Editor: Marcello Locatelli

Received: 17 May 2021

Accepted: 9 June 2021

Published: 29 June 2021

Publisher's Note: MDPI stays neutral with regard to jurisdictional claims in published maps and institutional affiliations.

Copyright: (C) 2021 by the authors. Licensee MDPI, Basel, Switzerland. This article is an open access article distributed under the terms and conditions of the Creative Commons Attribution (CC BY) license (https:// creativecommons.org/licenses/by/ $4.0 /)$.
Abstract: Polygoni Multiflori Radix Praeparata (PMRP), as the processed product of tuberous roots of Polygonum multiflorum Thunb., is one of the most famous traditional Chinese medicines, with a long history. However, in recent years, liver adverse reactions linked to PMRP have been frequently reported. Our work attempted to investigate the chemical constituents of PMRP for clinical research and safe medication. In this study, an effective and rapid method was established to separate and characterize the constituents in PMRP by combining ultra-high performance liquid chromatography with hybrid quadrupole-orbitrap mass spectrometry (UHPLC-Q-Exactive Orbitrap-MS). Based on the accurate mass measurements for molecular and characteristic fragment ions, a total of 103 compounds, including 24 anthraquinones, 21 stilbenes, 15 phenolic acids, 14 flavones, and 29 other compounds were identified or tentatively characterized. Forty-eight compounds were tentatively characterized from PMRP for the first time, and their fragmentation behaviors were summarized. There were 101 components in PMRP ethanol extract (PMRPE) and 91 components in PMRP water extract (PMRPW). Simultaneously, the peak areas of several potential xenobiotic components were compared in the detection, which showed that PMRPE has a higher content of anthraquinones and stilbenes. The obtained results can be used in pharmacological and toxicological research and provided useful information for further in vitro and in vivo studies.

Keywords: polygoni multiflori radix praeparata; UHPLC-Q-Exactive Orbitrap-MS; chemical constituents; traditional Chinese medicine

\section{Introduction}

Polygoni Multiflori Radix Praeparata (Zhiheshouwu in Chinese, PMRP), as a processed root of Polygonum multiflorum Thunb. (heshouwu in Chinese, PMR), has a long history in clinical application. The common processing method of PMRP is steaming or boiling PMR with a black bean decoction, as prescribed by the Chinese Pharmacopoeia. After processing, the concentrations of major components and traditional usage have changed. PMR contains more combined anthraquinones, while fewer stilbenes and more free anthraquinones are found in PMRP [1]. PMRP could enhance immune function, nourish the liver and kidney, prevent premature loss of hair, protect the nervous system, and inhibit atherosclerosis et al. [2-4]. Modern research has revealed that anthraquinones, stilbenes, flavonoids, and phenolic acids in PMRP are the major compounds of its pharmacological activities [5,6]. Several polyhydroxy stilbenes such as 2,3,5,4'-tetrahydroxystilbene-2-O$\beta$-D-glucoside(THSG) have a similar structure to resveratrol, and they have also been proven to have a strong ability to antioxidize and perform free radical scavenging activities [7]. Besides, THSG show great lipid-regulation and protection against neurotoxicity [8]. Anthraquinones are the major compounds with extensive activity, such as anti-tumor, antibacterial, and neuroprotective effects. Emodin induces neuronal differenti- 
ation through PI3K/Akt/GSK-3 $\beta$ pathways in Neuro2a cells [9]. Three anthraquinones, including physcion, emodin, and questin, were regarded as Cdc25B phosphatase inhibitors by strongly inhibiting the growth of human colon cancer cells [10]. Proanthocyanidins, isolated from MPRP, have the potential to be functional ingredients in reducing postprandial hyperglycemia, by inhibiting $\alpha$-amylase and $\alpha$-glucosidase [11].

However, with the widespread application of PMRP in the clinic, many adverse events of PMRP, including dyspnea, fever, rash, nephrotoxicity, and hepatotoxicity, have been reported in many countries such as Japan, China, Korea, Italy, Singapore, Spain, Australia, and the USA [12-14]. As the main organ of drug metabolism, the liver seems to be more susceptible to xenobiotic components. Therefore, the incidence of liver injury induced by PMRP has increased year by year [15]. Though some compounds of PMRP have positive physiological effects $[16,17]$, there have been many studies reporting that several xenobiotic compounds could induce idiosyncratic hepatotoxicity. Anthraquinones are generally assigned as the major compounds of xenobiotics, because other anthraquinone-containing herbal medicines were also reported to induce liver injury [18,19]. Constituents other than anthraquinones, such as stilbenes and phenolic acids, were also considered to have a major contribution to the idiosyncratic hepatotoxicity of PMRP [20]. To find the potentially xenobiotic components and mechanisms of hepatotoxicity, qualitative and quantitative research has been explored. Zhang et al. [21] reported that the emodin-8-O- $\beta$-D-glucoside (EG) could induce hepatotoxicity, and the combination of EG and THSG could cause more severe liver injury. Moreover, in previous literature, the THSG, physcion and emodin showed no, moderate and severe cytotoxicity, respectively [22]. Rhein, which has weaker toxicity than emodin, has been demonstrated to exert concentration- and time-dependent toxic effects on L-02 cells [23].

At present, only a few compounds have been explored in xenobiotic studies. Considering the multi-component and multi-target characteristics of traditional Chinese medicine, the chemical constituents of PMRP should be identified for further studies. Meanwhile, previous studies have suggested that PMRP, extracted with different extraction solvents, showed various degrees of liver injury, and the order of toxicity was described as PMRP ethanol extract (PMRPE) > PMRP water extract (PMRPW) [24,25]. Consequently, to identify and compare the different components between PMRPE and PMRPW, an effective and sensitive ultra-high performance liquid chromatography coupled with hybrid quadrupoleorbitrap mass spectrometry (UHPLC-Q-Exactive Orbitrap-MS) method was established for characterization of the constituents of them. The results of this investigation are meaningful, and would provide a material basis for further pharmacological and toxicological studies.

\section{Results and Discussion}

\subsection{Optimization of LC and MS Conditions}

LC conditions including mobile phase, flow rate, column, and column temperature were optimized to obtain a good separation and resolution. Compared with methanol, using acetonitrile as the organic phase showed stronger elutive power and detection sensitivity. Due to most compounds in PMRP contain carboxyl and phenolic hydroxyl, the addition of $0.1 \%$ formic acid in the phase system can obtain better mass spectrometric responses and improve the shapes of most peaks. Therefore, the mobile phase was acetonitrile (A)- $0.1 \%$ formic acid in water (B), with optimized gradient elution. The Waters HSS T3 column $(2.1 \mathrm{~mm} \times 100 \mathrm{~mm}, 1.8 \mu \mathrm{m}, \mathrm{UK})$ is suitable for the high polar compounds and high percentage of the aqueous phase, which have been applied to the characterization of the constituents of other botanical extracts. For the MS conditions, we chose the negative mode by comparing the intensity of compounds in both positive and negative modes. Meanwhile, according to the base peak intensity chromatograms (BPC), more compounds can be detected in the negative mode. Finally, other MS parameters were optimized to obtain high sensitivity for most compounds. The results indicated that the UHPLC-Q-Exactive Orbitrap-MS developed in this study is appropriate to detect the chemical constituents in PMRP. 


\subsection{Identification of the Chemical Constituents in PMRP}

An in-house database that includes chemical names, molecular formulas, accurate molecular mass, chemical structures, and relevant fragments was established by searching Science Direct of Elsevier, Chemspider, PubMed, and CNKI (Chinese National Knowledge Infrastructure). We used Xcalibur ${ }^{\mathrm{TM}}$ and TraceFinder to obtain accurate mass, elemental composition, and multiple-stage mass data. By matching the in-house database to compare and characterize the compounds in PMRPE and PMRPW, these formulas which have been reported in the literature can be considered. A total of 103 chemical constituents were tentatively represented, including 24 anthraquinones, 21 stilbenes, 15 phenolic acids, 14 flavones, and 29 other compounds. The base peak intensity chromatogram (BPC) is shown in Figures 1 and 2. The details of the identified compounds are summarized in Table 1 and the chemical structures of major constituents are shown in Figure S1.

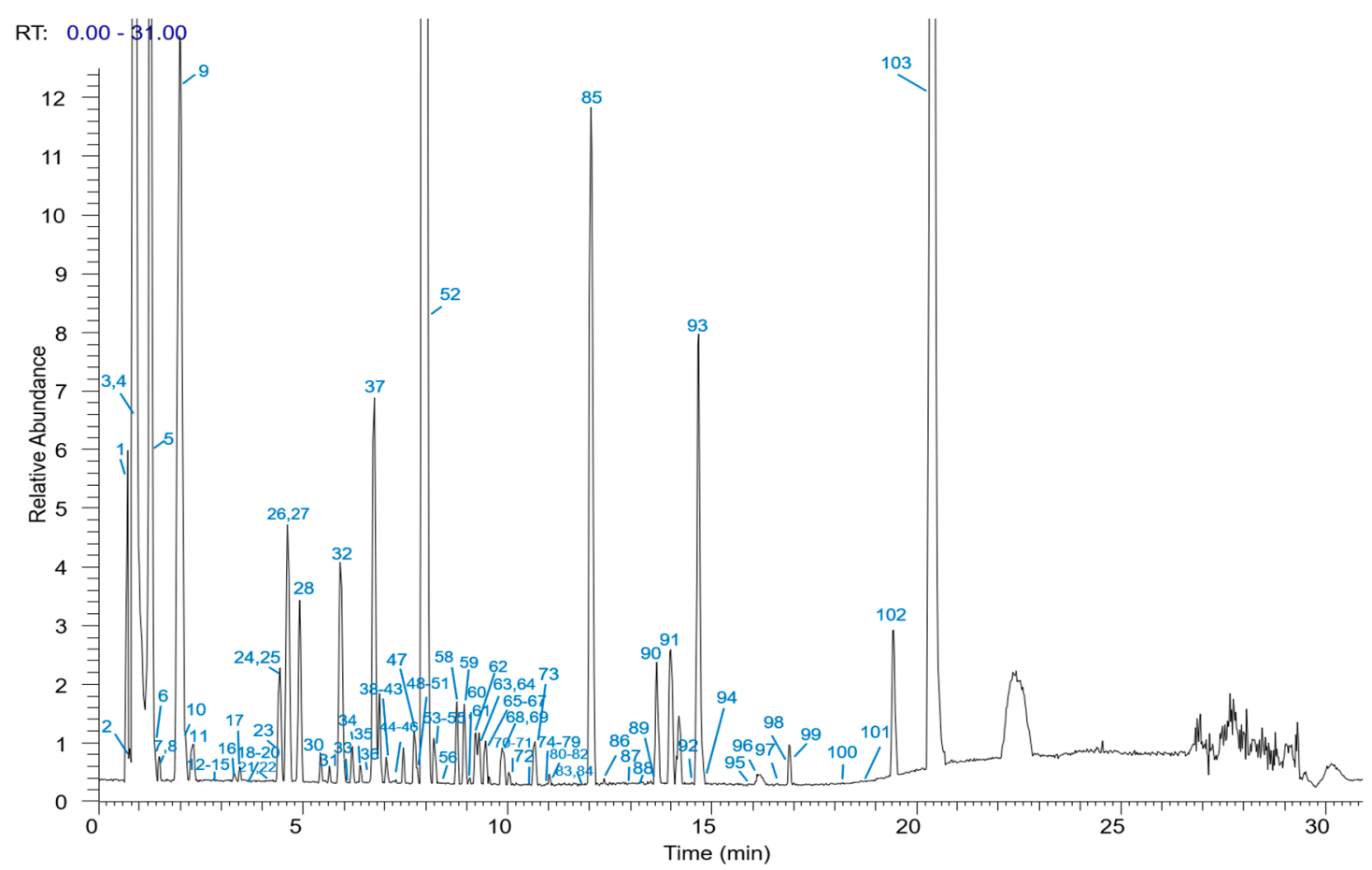

Figure 1. UHPLC-Q-Exactive Orbitrap-MS base peak intensity chromatogram (BPC) of PMRPE. 


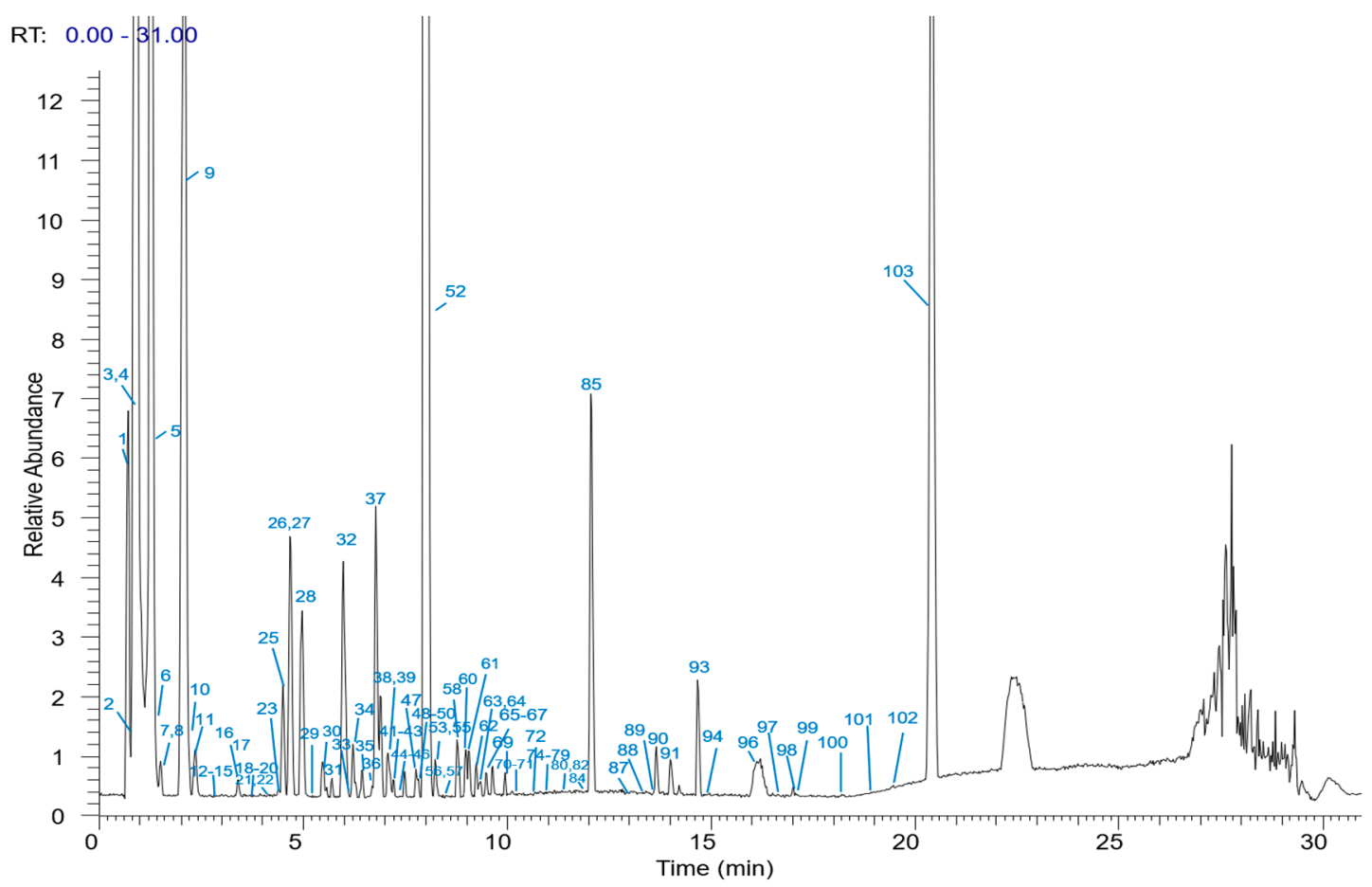

Figure 2. UHPLC-Q-Exactive Orbitrap-MS base peak intensity chromatogram (BPC) of PMRPW.

Table 1. Chemical constituents identified in PMRP by UHPLC-Q-Exactive Orbitrap-MS.

\begin{tabular}{|c|c|c|c|c|c|c|c|c|}
\hline NO. & $\begin{array}{l}\mathrm{RT} \\
(\mathrm{min})\end{array}$ & Identification & $\begin{array}{l}\text { Molecular } \\
\text { Formula }\end{array}$ & $\begin{array}{l}\text { Measured } \\
\text { Mass }[\mathrm{M}-\mathrm{H}]^{-}\end{array}$ & $\begin{array}{l}\text { Accuracy } \\
\text { Mass }[\mathbf{M}-\mathbf{H}]^{-}\end{array}$ & $\begin{array}{l}\text { Error } \\
(\mathrm{ppm})\end{array}$ & Characteristic Fragment Ions & Source \\
\hline \multicolumn{9}{|c|}{ Anthraquinones and derivatives } \\
\hline 30 & 5.88 & $\begin{array}{l}\text { Physcion-8-O-(6'-O- } \\
\text { malonyl)-hexose }\end{array}$ & $\mathrm{C}_{26} \mathrm{H}_{26} \mathrm{O}_{12}$ & 529.13391 & 529.13405 & -0.269 & $\begin{array}{l}\left.\text { 366.07205[M-H- } \mathrm{C}_{6} \mathrm{H}_{11} \mathrm{O}_{5}\right]^{-} \\
348.06122\left[\mathrm{M}-\mathrm{H}-\mathrm{C}_{6} \mathrm{H}_{11} \mathrm{O}_{5}-\mathrm{H}_{2} \mathrm{O}\right]^{-} \\
320.06482\left[\mathrm{M}-\mathrm{H}-\mathrm{C}_{6} \mathrm{H}_{11} \mathrm{O}_{5}-\mathrm{H}_{2} \mathrm{O}-\mathrm{CO}\right]^{-}\end{array}$ & $\begin{array}{l}\text { PMRPW, } \\
\text { PMRPE }\end{array}$ \\
\hline 33 & 6.09 & Rumejaposide $\mathrm{D}^{\mathrm{a}}$ & $\mathrm{C}_{21} \mathrm{H}_{22} \mathrm{O}_{11}$ & 449.10751 & 449.10784 & -0.730 & $\begin{array}{l}287.04864\left[\mathrm{M}-\mathrm{H}-\mathrm{C}_{6} \mathrm{H}_{10} \mathrm{O}_{5}\right]^{-} \\
269.04453\left[\mathrm{M}-\mathrm{H}-\mathrm{C}_{6} \mathrm{H}_{10} \mathrm{O}_{5}-\mathrm{H}_{2} \mathrm{O}\right]^{-} \\
259.06021\left[\mathrm{M}-\mathrm{H}-\mathrm{C}_{6} \mathrm{H}_{10} \mathrm{O}_{5}-\mathrm{CO}\right]^{-} \\
671.17426\left[\mathrm{M}-\mathrm{H}-\mathrm{C}_{6} \mathrm{H}_{10} \mathrm{O}_{5}\right]^{-}\end{array}$ & $\begin{array}{l}\text { PMRPW, } \\
\text { PMRPE }\end{array}$ \\
\hline 57 & 8.41 & Di-emodin-Di-glucoside $^{a}$ & $\mathrm{C}_{42} \mathrm{H}_{42} \mathrm{O}_{18}$ & 833.22919 & 833.22874 & 0.539 & $\begin{array}{l}509.12094\left[\mathrm{M}-\mathrm{H}-2 \mathrm{C}_{6} \mathrm{H}_{10} \mathrm{O}_{5}\right]^{-} \\
253.04974\left[\mathrm{M}-\mathrm{H}-2 \mathrm{C}_{6} \mathrm{H}_{10} \mathrm{O}_{5-}^{-}\right. \\
\left.\mathrm{C}_{15} \mathrm{H}_{12} \mathrm{O}_{4}\right]^{-}\end{array}$ & PMRPW \\
\hline 58 & 8.86 & $\begin{array}{l}\text { Isomer emodin-8-O- }\left(6^{\prime}-O-\right. \\
\text { acetyl)- } \beta \text {-D- } \\
\text { glucoside }\end{array}$ & $\mathrm{C}_{23} \mathrm{H}_{22} \mathrm{O}_{11}$ & 473.10638 & 473.10784 & -3.081 & $\begin{array}{l}311.05423\left[\mathrm{M}-\mathrm{H}-\mathrm{C}_{6} \mathrm{H}_{10} \mathrm{O}_{5}\right]^{-} \\
283.06085\left[\mathrm{M}-\mathrm{H}-\mathrm{C}_{6} \mathrm{H}_{10} \mathrm{O}_{5}-\mathrm{CO}\right]^{-} \\
255.06544\left[\mathrm{M}-\mathrm{H}-\mathrm{C}_{6} \mathrm{H}_{10} \mathrm{O}_{5}-2 \mathrm{CO}\right]^{-}\end{array}$ & $\begin{array}{l}\text { PMRPW, } \\
\text { PMRPE }\end{array}$ \\
\hline 77 & 10.83 & Citreorosein- $O$-glucoside $^{a}$ & $\mathrm{C}_{21} \mathrm{H}_{20} \mathrm{O}_{11}$ & 447.09048 & 447.09219 & -3.820 & $\begin{array}{l}300.02811\left[\mathrm{M}-\mathrm{H}-\mathrm{C}_{6} \mathrm{H}_{10} \mathrm{O}_{4}\right]^{-} \\
268.03757\left[\mathrm{M}-\mathrm{H}-\mathrm{C}_{6} \mathrm{H}_{10} \mathrm{O}_{4}-2 \mathrm{O}\right]^{-} \\
240.04250\left[\mathrm{M}-\mathrm{H}-\mathrm{C}_{6} \mathrm{H}_{10} \mathrm{O}_{4}-\mathrm{CO}\right]^{-}\end{array}$ & $\begin{array}{l}\text { PMRPW, } \\
\text { PMRPE }\end{array}$ \\
\hline 78 & 10.95 & Chrysophanol $^{\text {b }}$ & $\mathrm{C}_{15} \mathrm{H}_{10} \mathrm{O}_{4}$ & 253.05020 & 253.04954 & 2.627 & $\begin{array}{l}225.05373[\mathrm{M}-\mathrm{H}-\mathrm{CO}]^{-} \\
197.56078[\mathrm{M}-\mathrm{H}-2 \mathrm{CO}]^{-} \\
181.06459\left[\mathrm{M}-\mathrm{H}-\mathrm{CO}_{2}-\mathrm{CO}\right]^{-} \\
311.05438\left[\mathrm{M}-\mathrm{H}-\mathrm{C}_{6} \mathrm{H}_{10} \mathrm{O}_{5}\right]^{-}\end{array}$ & $\begin{array}{l}\text { PMRPW, } \\
\text { PMRPE }\end{array}$ \\
\hline 84 & 11.97 & $\begin{array}{l}\text { 2-Acetylemodin-8-O- } \beta \text {-D- } \\
\text { glucoside }\end{array}$ & $\mathrm{C}_{23} \mathrm{H}_{22} \mathrm{O}_{11}$ & 473.10583 & 473.10784 & -4.424 & $\begin{array}{l}311.05438\left[\mathrm{M}-\mathrm{H}-\mathrm{C}_{6} \mathrm{H}_{10} \mathrm{O}_{5}\right]^{-} \\
269.04575\left[\mathrm{M}-\mathrm{H}_{-}-\mathrm{C}_{6} \mathrm{H}_{10} \mathrm{O}_{5}-\mathrm{C}_{2} \mathrm{H}_{2} \mathrm{O}\right]^{-} \\
241.04889\left[\mathrm{M}-\mathrm{H}-\mathrm{C}_{6} \mathrm{H}_{10} \mathrm{O}_{5}-\mathrm{C}_{2} \mathrm{H}_{2} \mathrm{O}-\right. \\
\mathrm{CO}^{-}\end{array}$ & $\begin{array}{l}\text { PMRPW, } \\
\text { PMRPE }\end{array}$ \\
\hline 85 & 12.12 & Emodin-8-O- $\beta$-D-glucoside ${ }^{b}$ & $\mathrm{C}_{21} \mathrm{H}_{20} \mathrm{O}_{10}$ & 431.09637 & 431.09727 & -2.095 & $\begin{array}{l}269.04520\left[\mathrm{M}-\mathrm{H}-\mathrm{C}_{6} \mathrm{H}_{10} \mathrm{O}_{5}\right]^{-} \\
241.04926\left[\mathrm{M}-\mathrm{H}-\mathrm{C}_{6} \mathrm{H}_{10} \mathrm{O}_{5}-\mathrm{CO}\right]^{-} \\
225.05426\left[\mathrm{M}-\mathrm{H}-\mathrm{C}_{6} \mathrm{H}_{10} \mathrm{O}_{5}-\mathrm{CO}_{2}\right]^{-}\end{array}$ & $\begin{array}{l}\text { PMRPW, } \\
\text { PMRPE }\end{array}$ \\
\hline 86 & 12.35 & $\begin{array}{l}\text { Emodin-O-glucoside- } \\
\text { gallate }^{\text {a }}\end{array}$ & $\mathrm{C}_{28} \mathrm{H}_{24} \mathrm{O}_{14}$ & 583.10736 & 583.10823 & -0.872 & $\begin{array}{l}269.04562\left[\mathrm{M}-\mathrm{H}-\mathrm{C}_{6} \mathrm{H}_{10} \mathrm{O}_{5}-\mathrm{C}_{7} \mathrm{H}_{4} \mathrm{O}_{4}\right]^{-} \\
225.05466\left[\mathrm{M}-\mathrm{H}-\mathrm{C}_{6} \mathrm{H}_{10} \mathrm{O}_{5}-\mathrm{C}_{7} \mathrm{H}_{4} \mathrm{O}_{4^{-}}\right. \\
\left.\mathrm{CO}_{2}\right]^{-}\end{array}$ & PMRPE \\
\hline 87 & 13.00 & 6-Carboxyl emodin ${ }^{a}$ & $\mathrm{C}_{16} \mathrm{H}_{10} \mathrm{O}_{7}$ & 313.03448 & 313.03428 & 0.642 & $\begin{array}{l}269.04544\left[\mathrm{M}-\mathrm{H}-\mathrm{CO}_{2}\right]^{-} \\
241.05034\left[\mathrm{M}-\mathrm{H}-\mathrm{CO}_{2}-\mathrm{CO}\right]^{-} \\
225.95458\left[\mathrm{M}-\mathrm{H}-2 \mathrm{CO}_{2}\right]^{-}\end{array}$ & $\begin{array}{l}\text { PMRPW, } \\
\text { PMRPE }\end{array}$ \\
\hline 89 & 13.54 & Physcion-8-O- $\beta$-D-glucoside & $\mathrm{C}_{22} \mathrm{H}_{22} \mathrm{O}_{10}$ & 445.11273 & 445.11292 & -0.434 & $\begin{array}{l}283.06104\left[\mathrm{M}-\mathrm{H}-\mathrm{C}_{6} \mathrm{H}_{10} \mathrm{O}_{5}\right]^{-} \\
255.06458\left[\mathrm{M}-\mathrm{H}-\mathrm{C}_{6} \mathrm{H}_{10} \mathrm{O}_{5}-\mathrm{CO}\right]^{-} \\
239.06963\left[\mathrm{M}-\mathrm{H}-\mathrm{C}_{6} \mathrm{H}_{10} \mathrm{O}_{5}-\mathrm{CO}_{2}\right]^{-}\end{array}$ & $\begin{array}{l}\text { PMRPW, } \\
\text { PMRPE }\end{array}$ \\
\hline 90 & 13.65 & Physcion ${ }^{b}$ & $\mathrm{C}_{16} \mathrm{H}_{12} \mathrm{O}_{5}$ & 283.06113 & 283.06010 & 3.639 & $\begin{array}{l}268.03760\left[\mathrm{M}-\mathrm{H}-\mathrm{CH}_{3}\right]^{-} \\
240.04179\left[\mathrm{M}-\mathrm{H}-\mathrm{CH}_{3}-\mathrm{CO}\right]^{-} \\
212.04668\left[\mathrm{M}-\mathrm{H}-\mathrm{CH}_{3}-2 \mathrm{CO}\right]^{-}\end{array}$ & $\begin{array}{l}\text { PMRPW, } \\
\text { PMRPE }\end{array}$ \\
\hline
\end{tabular}


Table 1. Cont.

\begin{tabular}{|c|c|c|c|c|c|c|c|c|}
\hline NO. & $\begin{array}{l}\text { RT } \\
(\mathrm{min})\end{array}$ & Identification & $\begin{array}{l}\text { Molecular } \\
\text { Formula }\end{array}$ & $\begin{array}{l}\text { Measured } \\
\text { Mass }[\mathbf{M}-\mathbf{H}]^{-}\end{array}$ & $\begin{array}{l}\text { Accuracy } \\
\text { Mass }[\mathbf{M}-\mathbf{H}]^{-}\end{array}$ & $\begin{array}{l}\text { Error } \\
(\text { ppm) }\end{array}$ & Characteristic Fragment Ions & Source \\
\hline 91 & 14.09 & Citreorosein & $\mathrm{C}_{1} 5 \mathrm{H}_{10} \mathrm{O}_{6}$ & 285.04041 & 285.03936 & 3.668 & $\begin{array}{l}257.04422[\mathrm{M}-\mathrm{H}-\mathrm{CO}]^{-} \\
241.04965\left[\mathrm{M}-\mathrm{H}-\mathrm{CO}_{2}\right]^{-} \\
227\left[\mathrm{M}-\mathrm{H}-\mathrm{CO}-\mathrm{CH}_{2} \mathrm{O}\right]^{-}\end{array}$ & $\begin{array}{l}\text { PMRPW, } \\
\text { PMRPE }\end{array}$ \\
\hline 92 & 14.59 & Chrysophanol anthrone ${ }^{a}$ & $\mathrm{C}_{15} \mathrm{H}_{12} \mathrm{O}_{3}$ & 239.07027 & 239.06989 & -1.593 & $\begin{array}{l}\text { 210.89307[M-H-CO }]^{-} \\
182.89648[\mathrm{M}-\mathrm{H}-2 \mathrm{CO}]^{-}\end{array}$ & PMRPE \\
\hline 93 & 14.69 & Questinol & $\mathrm{C}_{16} \mathrm{H}_{12} \mathrm{O}_{6}$ & 299.05493 & 299.05501 & -0.283 & $\begin{array}{l}268.03696\left[\mathrm{M}-\mathrm{H}-\mathrm{CH}_{3} \mathrm{O}\right]^{-} \\
253.04982\left[\mathrm{M}-\mathrm{H}-\mathrm{CH}_{3} \mathrm{O}-\mathrm{CH}_{3}\right]^{-} \\
240.04204\left[\mathrm{M}-\mathrm{H}-\mathrm{CH}_{3} \mathrm{O}-\mathrm{CO}\right]^{-}\end{array}$ & $\begin{array}{l}\text { PMRPW, } \\
\text { PMRPE }\end{array}$ \\
\hline 94 & 14.87 & Hydroxyl-rhein ${ }^{a}$ & $\mathrm{C}_{15} \mathrm{H}_{8} \mathrm{O}_{7}$ & 299.01867 & 299.01863 & 0.070 & $\begin{array}{l}\text { 255.02777[M-H-CO }{ }_{2}^{-} \\
227.03313\left[\mathrm{M}-\mathrm{H}-\mathrm{CO}_{2}-\mathrm{CO}\right]^{-} \\
199.03928\left[\mathrm{M}-\mathrm{H}-\mathrm{CO}_{2}-2 \mathrm{CO}\right]^{-}\end{array}$ & $\begin{array}{l}\text { PMRPW, } \\
\text { PMRPE }\end{array}$ \\
\hline 95 & 15.94 & Digitolutein $^{\text {a }}$ & $\mathrm{C}_{16} \mathrm{H}_{12} \mathrm{O}_{4}$ & 267.06601 & 267.06519 & 3.088 & $\begin{array}{l}224.04675\left[\mathrm{M}-\mathrm{H}-\mathrm{CO}-\mathrm{CH}_{3}\right]^{-} \\
220.53163\left[\mathrm{M}-\mathrm{H}-\mathrm{CO}-\mathrm{H}_{2} \mathrm{O}^{-}\right. \\
149.02373\left[\mathrm{M}-\mathrm{H}-2 \mathrm{CO}-\mathrm{CH}_{2} \mathrm{O}-\mathrm{CH}_{2}-\right. \\
\mathrm{H}_{2} \mathrm{O}^{-}\end{array}$ & PMRPE \\
\hline 96 & 16.13 & Isomer Citreorosein & $\mathrm{C}_{15} \mathrm{H}_{10} \mathrm{O}_{6}$ & 285.04041 & 285.03936 & 3.668 & $\begin{array}{l}257.04462\left[\mathrm{M}-\mathrm{H}-\mathrm{CO}^{-}\right. \\
241.04955\left[\mathrm{M}-\mathrm{H}-\mathrm{CO}_{2}\right]^{-} \\
211.03883\left[\mathrm{M}-\mathrm{H}-\mathrm{CO}_{2}-\mathrm{CH}_{2} \mathrm{O}\right]^{-}\end{array}$ & $\begin{array}{l}\text { PMRPW, } \\
\text { PMRPE }\end{array}$ \\
\hline 98 & 17.02 & Emodin anthrone $^{\mathrm{a}}$ & $\mathrm{C}_{15} \mathrm{H}_{12} \mathrm{O}_{4}$ & 255.06572 & 255.06519 & 2.096 & $\begin{array}{l}240.04176\left[\mathrm{M}-\mathrm{H}-\mathrm{CH}_{3}\right]^{-} \\
225.05334\left[\mathrm{M}-\mathrm{H}-\mathrm{CH}_{2} \mathrm{O}\right]^{-} \\
212.04684\left[\mathrm{M}-\mathrm{H}-\mathrm{CH}_{3}-\mathrm{CO}\right]^{-}\end{array}$ & $\begin{array}{l}\text { PMRPW, } \\
\text { PMRPE }\end{array}$ \\
\hline 99 & 17.05 & Isomer physcion & $\mathrm{C}_{16} \mathrm{H}_{12} \mathrm{O}_{5}$ & 283.06067 & 283.06010 & 2.014 & $\begin{array}{l}240.04173\left[\mathrm{M}-\mathrm{H}-\mathrm{CH}_{3}-\mathrm{CO}\right]^{-} \\
268.03635\left[\mathrm{M}-\mathrm{H}-\mathrm{CH}_{3}\right]^{-}\end{array}$ & $\begin{array}{l}\text { PMRPW, } \\
\text { PMRPE }\end{array}$ \\
\hline 100 & 18.20 & $\begin{array}{l}\text { Emodin-3- } \\
\text { ethyl ether }\end{array}$ & $\mathrm{C}_{17} \mathrm{H}_{14} \mathrm{O}_{5}$ & 297.07452 & 297.07575 & 0.606 & $\begin{array}{l}282.05472\left[\mathrm{M}-\mathrm{H}-\mathrm{CH}_{3}\right]^{-} \\
269.08282[\mathrm{M}-\mathrm{H}-\mathrm{CO}]^{-} \\
254.05786\left[\mathrm{M}-\mathrm{H}-\mathrm{C}_{2} \mathrm{H}_{5}-\mathrm{CH}_{2}\right]^{-}\end{array}$ & $\begin{array}{l}\text { PMRPW, } \\
\text { PMRPE }\end{array}$ \\
\hline 101 & 18.86 & 2-Acetylemodin & $\mathrm{C}_{17} \mathrm{H}_{12} \mathrm{O}_{6}$ & 311.05408 & 311.05501 & -3.004 & $\begin{array}{l}296.03165\left[\mathrm{M}-\mathrm{H}-\mathrm{CH}_{3}\right]^{-} \\
283.06100[\mathrm{M}-\mathrm{H}-\mathrm{CO}]^{-} \\
269.04504\left[\mathrm{M}-\mathrm{H}-\mathrm{C}_{2} \mathrm{H}_{2} \mathrm{O}\right]^{-}\end{array}$ & $\begin{array}{l}\text { PMRPW, } \\
\text { PMRPE }\end{array}$ \\
\hline 102 & 19.52 & Isomer 2-acetylemodin & $\mathrm{C}_{17} \mathrm{H}_{12} \mathrm{O}_{6}$ & 311.05426 & 311.05501 & -2.426 & $\begin{array}{l}283.06119[\mathrm{M}-\mathrm{H}-\mathrm{CO}]^{-} \\
269.06583\left[\mathrm{M}-\mathrm{H}-\mathrm{C}_{2} \mathrm{H}_{2} \mathrm{O}\right]^{-} \\
240.04160\left[\mathrm{M}-\mathrm{H}-2 \mathrm{CO}-\mathrm{CH}_{3}\right]^{-}\end{array}$ & $\begin{array}{l}\text { PMRPW, } \\
\text { PMRPE }\end{array}$ \\
\hline 103 & 20.37 & Emodin $^{b}$ & $\mathrm{C}_{15} \mathrm{H}_{10} \mathrm{O}_{5}$ & 269.04514 & 269.04445 & 2.565 & $\begin{array}{l}241.04941[\mathrm{M}-\mathrm{H}-\mathrm{CO}]^{-} \\
213.05467[\mathrm{M}-\mathrm{H}-2 \mathrm{CO}]^{-} \\
225.05450\left[\mathrm{M}-\mathrm{H}-\mathrm{CO}_{2}\right]^{-}\end{array}$ & $\begin{array}{l}\text { PMRPW, } \\
\text { PMRPE }\end{array}$ \\
\hline \multicolumn{9}{|c|}{ Stilbenes and derivatives } \\
\hline 29 & 5.24 & Polygonumosides C & $\mathrm{C}_{40} \mathrm{H}_{44} \mathrm{O}_{19}$ & 827.23828 & 827.23931 & -1.239 & $\begin{array}{l}421.11276\left[\mathrm{M}-\mathrm{H}-\mathrm{C}_{20} \mathrm{H}_{22} \mathrm{O}_{9}\right]^{-} \\
259.06009\left[\mathrm{M}-\mathrm{H}-\mathrm{C}_{20} \mathrm{H}_{22} \mathrm{O}_{9}-\mathrm{C}_{6} \mathrm{H}_{10} \mathrm{O}_{5}\right]^{-} \\
241.04927\left[\mathrm{M}-\mathrm{H}-\mathrm{C}_{20} \mathrm{H}_{22} \mathrm{O}_{9^{-}}\right. \\
\left.\mathrm{C}_{6} \mathrm{H}_{10} \mathrm{O}_{5}-\mathrm{H}_{2} \mathrm{O}\right]^{-}\end{array}$ & PMRPW \\
\hline 37 & 6.69 & $\begin{array}{l}\text { Isomer } \\
3,4,5,4^{\prime} \text {-tetrahydroxystilbene }\end{array}$ & $\mathrm{C}_{14} \mathrm{H}_{12} \mathrm{O}_{4}$ & 243.06465 & 243.06519 & -2.202 & $\begin{array}{l}225.05467\left[\mathrm{M}-\mathrm{H}-\mathrm{H}_{2} \mathrm{O}\right]^{-} \\
215.06952[\mathrm{M}-\mathrm{H}-\mathrm{CO}]^{-} \\
197.05991\left[\mathrm{M}-\mathrm{H}-\mathrm{H}_{2} \mathrm{O}-\mathrm{CO}\right]^{-}\end{array}$ & $\begin{array}{l}\text { PMRPW, } \\
\text { PMRPE }\end{array}$ \\
\hline 41 & 7.07 & Rhapontin $^{a}$ & $\mathrm{C}_{21} \mathrm{H}_{24} \mathrm{O}_{9}$ & 419.13242 & 419.13366 & -2.955 & $\begin{array}{l}\left.\text { 257.07297[M-H- } \mathrm{C}_{6} \mathrm{H}_{10} \mathrm{O}_{5}\right]^{-} \\
239.03387\left[\mathrm{M}-\mathrm{H}-\mathrm{C}_{6} \mathrm{H}_{10} \mathrm{O}_{5}-\mathrm{H}_{2} \mathrm{O}\right]^{-} \\
227.03279\left[\mathrm{M}-\mathrm{H}-\mathrm{C}_{6} \mathrm{H}_{10} \mathrm{O}_{5}-\mathrm{CH}_{2} \mathrm{O}\right]^{-}\end{array}$ & $\begin{array}{l}\text { PMRPW, } \\
\text { PMRPE }\end{array}$ \\
\hline 44 & 7.31 & $\begin{array}{l}\text { Tetrahydroxystilbene-O-di- } \\
\text { glucoside }\end{array}$ & $\mathrm{C}_{26} \mathrm{H}_{32} \mathrm{O}_{14}$ & 567.17004 & 567.17083 & -1.396 & $\begin{array}{l}\left.\text { 243.06516[M-H-2 } \mathrm{C}_{6} \mathrm{H}_{10} \mathrm{O}_{5}\right]^{-} \\
225.05428\left[\mathrm{M}-\mathrm{H}-2 \mathrm{C}_{6} \mathrm{H}_{10} \mathrm{O}_{5}-\mathrm{H}_{2} \mathrm{O}\right]^{-} \\
\left.\text {197.06007[M-H-2 } \mathrm{C}_{6} \mathrm{H}_{10} \mathrm{O}_{5}-\mathrm{H}_{2} \mathrm{O}-\mathrm{CO}\right]^{-}\end{array}$ & $\begin{array}{l}\text { PMRPW, } \\
\text { PMRPE }\end{array}$ \\
\hline 45 & 7.38 & $\begin{array}{l}\beta \text {-D-glucoside,4-[2,3- } \\
\text { dihydro-3-(hydroxymethyl)- } \\
\text { 5-(3-hydroxypropyl)-7- } \\
\text { methoxy-2-yl]-2- } \\
\text { methoxyphenyl }^{\text {a }}\end{array}$ & $\mathrm{C}_{26} \mathrm{H}_{34} \mathrm{O}_{11}$ & 521.20062 & 521.20174 & -2.145 & $\begin{array}{l}359.147229\left[\mathrm{M}-\mathrm{H}-\mathrm{C}_{6} \mathrm{H}_{10} \mathrm{O}_{5}\right]^{-} \\
313.10608\left[\mathrm{M}-\mathrm{H}-\mathrm{C}_{6} \mathrm{H}_{10} \mathrm{O}_{5}-\mathrm{H}_{2} \mathrm{O}-\mathrm{CO}\right]^{-}\end{array}$ & $\begin{array}{l}\text { PMRPW, } \\
\text { PMRPE }\end{array}$ \\
\hline 47 & 7.76 & Resveratrol $^{\mathrm{a}}$ & $\mathrm{C}_{14} \mathrm{H}_{12} \mathrm{O}_{3}$ & 227.07057 & 227.07027 & 1.318 & $\begin{array}{l}\left.\text { 185.05931[M-H-H } \mathrm{H}_{2} \mathrm{O}-\mathrm{CH}_{2}\right]^{-} \\
170.97986\left[\mathrm{M}-\mathrm{H}-\mathrm{H}_{2} \mathrm{O}-2 \mathrm{CH}_{2}\right]^{-} \\
143.04947\left[\mathrm{M}-\mathrm{H}-\mathrm{C}_{4} \mathrm{H}_{4} \mathrm{O}_{2}\right]^{-}\end{array}$ & $\begin{array}{l}\text { PMRPW, } \\
\text { PMRPE }\end{array}$ \\
\hline 48 & 7.67 & $\begin{array}{l}\text { Isomer } \\
\text { Tetrahydroxystilbene-O-di- } \\
\text { glucoside }\end{array}$ & $\mathrm{C}_{26} \mathrm{H}_{32} \mathrm{O}_{14}$ & 567.17059 & 567.17083 & -0.427 & $\begin{array}{l}243.06509\left[\mathrm{M}-\mathrm{H}-2 \mathrm{C}_{6} \mathrm{H}_{10} \mathrm{O}_{5}\right]^{-} \\
225.05261\left[\mathrm{M}-\mathrm{H}-2 \mathrm{C}_{6} \mathrm{H}_{10} \mathrm{O}_{5}-\mathrm{H}_{2} \mathrm{O}\right]^{-}\end{array}$ & $\begin{array}{l}\text { PMRPW, } \\
\text { PMRPE }\end{array}$ \\
\hline 50 & 7.89 & $3,4,5,4^{\prime}$-Tetrahydroxystilbene & $\mathrm{C}_{14} \mathrm{H}_{12} \mathrm{O}_{4}$ & 243.06517 & 243.06519 & -0.063 & $\begin{array}{l}225.05469\left[\mathrm{M}-\mathrm{H}-\mathrm{H}_{2} \mathrm{O}\right]^{-} \\
197.05965\left[\mathrm{M}-\mathrm{H}-\mathrm{H}_{2} \mathrm{O}-\mathrm{CO}\right]^{-} \\
169.06514\left[\mathrm{M}-\mathrm{H}-\mathrm{H}_{2} \mathrm{O}-2 \mathrm{CO}\right]^{-}\end{array}$ & $\begin{array}{l}\text { PMRPW, } \\
\text { PMRPE }\end{array}$ \\
\hline 52 & 7.94 & $\begin{array}{l}2,3,5, \\
4^{\prime} \text {-Tetrahydroxystilbene-2-O- } \\
\beta \text {-D-glucoside }{ }^{\mathrm{b}}\end{array}$ & $\mathrm{C}_{20} \mathrm{H}_{22} \mathrm{O}_{9}$ & 405.11670 & 405.11801 & -0.885 & $\begin{array}{l}\left.\text { 243.06503[M-H- } \mathrm{C}_{6} \mathrm{H}_{10} \mathrm{O}_{5}\right]^{-} \\
225.05450\left[\mathrm{M}-\mathrm{H}-\mathrm{C}_{6} \mathrm{H}_{10} \mathrm{O}_{5}-\mathrm{H}_{2} \mathrm{O}\right]^{-} \\
215.07039\left[\mathrm{M}-\mathrm{H}-\mathrm{C}_{6} \mathrm{H}_{10} \mathrm{O}_{5}-\mathrm{CO}\right]^{-}\end{array}$ & $\begin{array}{l}\text { PMRPW, } \\
\text { PMRPE }\end{array}$ \\
\hline 56 & 8.32 & Multiflorumisides $\mathrm{A}^{\mathrm{a}}$ & $\mathrm{C}_{40} \mathrm{H}_{44} \mathrm{O}_{18}$ & 811.24438 & 811.24439 & -0.013 & $\begin{array}{l}\left.\text { 649.19263[M-H- } \mathrm{C}_{6} \mathrm{H}_{10} \mathrm{O}_{5}\right]^{-} \\
405.11447\left[\mathrm{M}-\mathrm{H}-\mathrm{C}_{20} \mathrm{H}_{22} \mathrm{O}_{9}\right]^{-} \\
243.06512\left[\mathrm{M}-\mathrm{H}-\mathrm{C}_{20} \mathrm{H}_{22} \mathrm{O}_{9}-\mathrm{C}_{6} \mathrm{H}_{10} \mathrm{O}_{5}\right]^{-}\end{array}$ & $\begin{array}{l}\text { PMRPW, } \\
\text { PMRPE }\end{array}$ \\
\hline 61 & 9.17 & Polygonumoside A & $\mathrm{C}_{27} \mathrm{H}_{24} \mathrm{O}_{13}$ & 555.11487 & 555.11332 & 2.797 & $\begin{array}{l}\left.\text { 393.05923[M-H-C } \mathrm{C}_{6} \mathrm{H}_{10} \mathrm{O}_{5}\right]^{-} \\
349.07019\left[\mathrm{M}-\mathrm{H}-\mathrm{C}_{6} \mathrm{H}_{10} \mathrm{O}_{5}-\mathrm{CO}_{2}\right]^{-} \\
300.99774\left[\mathrm{M}-\mathrm{H}-\mathrm{C}_{6} \mathrm{H}_{10} \mathrm{O}_{5}-\mathrm{C}_{6} \mathrm{H}_{4} \mathrm{O}\right]^{-}\end{array}$ & $\begin{array}{l}\text { PMRPW, } \\
\text { PMRPE }\end{array}$ \\
\hline 64 & 9.53 & Isomer polygonumoside A & $\mathrm{C}_{27} \mathrm{H}_{24} \mathrm{O}_{13}$ & 555.11432 & 555.11332 & 1.807 & $\begin{array}{l}\left.\text { 393.05942[M-H- } \mathrm{C}_{6} \mathrm{H}_{10} \mathrm{O}_{5}\right]^{-} \\
349.06873\left[\mathrm{M}-\mathrm{H}-\mathrm{C}_{6} \mathrm{H}_{10} \mathrm{O}_{5}-\mathrm{CO}_{2}\right]^{-} \\
300.99670\left[\mathrm{M}-\mathrm{H}-\mathrm{C}_{6} \mathrm{H}_{10} \mathrm{O}_{5}-\mathrm{C}_{6} \mathrm{H}_{4} \mathrm{O}\right]^{-}\end{array}$ & $\begin{array}{l}\text { PMRPW, } \\
\text { PMRPE }\end{array}$ \\
\hline
\end{tabular}


Table 1. Cont.

\begin{tabular}{|c|c|c|c|c|c|c|c|c|}
\hline NO. & $\begin{array}{l}\text { RT } \\
(\mathrm{min})\end{array}$ & Identification & $\begin{array}{l}\text { Molecular } \\
\text { Formula }\end{array}$ & $\begin{array}{l}\text { Measured } \\
\text { Mass }[\mathrm{M}-\mathrm{H}]^{-}\end{array}$ & $\begin{array}{l}\text { Accuracy } \\
\text { Mass }[\mathbf{M}-\mathbf{H}]^{-}\end{array}$ & $\begin{array}{l}\text { Error } \\
(\mathrm{ppm})\end{array}$ & Characteristic Fragment Ions & Source \\
\hline 66 & 9.56 & $\begin{array}{l}2,3,5,4^{\prime} \text { - } \\
\text { Tetrahydroxystilbene-O- } \\
\text { (malonyl)- } \\
\beta \text {-D-glucoside }\end{array}$ & $\mathrm{C}_{23} \mathrm{H}_{24} \mathrm{O}_{12}$ & 491.11929 & 491.11840 & 1.807 & $\begin{array}{l}329.09622\left[\mathrm{M}-\mathrm{H}-\mathrm{C}_{6} \mathrm{H}_{10} \mathrm{O}_{5}\right]^{-} \\
313.03226\left[\mathrm{M}-\mathrm{H}-\mathrm{C}_{6} \mathrm{H}_{10} \mathrm{O}_{5}-\mathrm{H}_{2} \mathrm{O}\right]^{-} \\
285.04071\left[\mathrm{M}-\mathrm{H}-\mathrm{C}_{6} \mathrm{H}_{10} \mathrm{O}_{5}-\mathrm{H}_{2} \mathrm{O}-\mathrm{CO}\right]^{-}\end{array}$ & $\begin{array}{l}\text { PMRPW, } \\
\text { PMRPE }\end{array}$ \\
\hline 67 & 9.57 & $\begin{array}{l}\text { Tetrahydroxystilbene-O- } \\
\text { (galloyl)-glucoside }\end{array}$ & $\mathrm{C}_{27} \mathrm{H}_{26} \mathrm{O}_{13}$ & 557.12933 & 557.12897 & 0.651 & $\begin{array}{l}405.05499\left[\mathrm{M}-\mathrm{H}-\mathrm{C}_{7} \mathrm{H}_{4} \mathrm{O}_{4}\right]^{-} \\
243.06503\left[\mathrm{M}-\mathrm{H}-\mathrm{C}_{7} \mathrm{H}_{4} \mathrm{O}_{4}-\mathrm{C}_{6} \mathrm{H}_{10} \mathrm{O}_{5}\right]^{-} \\
225.05434\left[\mathrm{M}-\mathrm{H}-\mathrm{C}_{7} \mathrm{H}_{4} \mathrm{O}_{4}-\mathrm{C}_{6} \mathrm{H}_{10} \mathrm{O}_{5^{-}}\right. \\
\left.\mathrm{H}_{2} \mathrm{O}\right]^{-}\end{array}$ & $\begin{array}{l}\text { PMRPW, } \\
\text { PMRPE }\end{array}$ \\
\hline 68 & 9.87 & $\begin{array}{l}2,3,5,4^{\prime}- \\
\text { Tetrahydroxystilbene-2-O- } \\
\left(2^{\prime \prime}-O \text {-acetyl)- } \beta \text {-D-glucoside }\right.\end{array}$ & $\mathrm{C}_{22} \mathrm{H}_{24} \mathrm{O}_{10}$ & 447.12930 & 447.12857 & 1.625 & $\begin{array}{l}243.06511\left[\mathrm{M}-\mathrm{H}-\mathrm{C}_{6} \mathrm{H}_{10} \mathrm{O}_{5}-\mathrm{C}_{2} \mathrm{H}_{2} \mathrm{O}\right]^{-} \\
225.05455\left[\mathrm{M}-\mathrm{H}-\mathrm{C}_{6} \mathrm{H}_{10} \mathrm{O}_{5}-\mathrm{C}_{2} \mathrm{H}_{2} \mathrm{O}-\right. \\
\left.\mathrm{H}_{2} \mathrm{O}\right]^{-} \\
284.08289\left[\mathrm{M}-\mathrm{H}-\mathrm{C}_{6} \mathrm{H}_{11} \mathrm{O}_{5}\right]^{-}\end{array}$ & PMRPE \\
\hline 69 & 9.95 & $\begin{array}{l}\text { Piceatannol-3-O- } \beta-\mathrm{D}-\left(6^{\prime \prime}-\mathrm{O}-\right. \\
\text { galloyl)- } \\
\text { glucoside }\end{array}$ & $\mathrm{C}_{27} \mathrm{H}_{26} \mathrm{O}_{13}$ & 557.12817 & 557.12897 & -1.431 & $\begin{array}{l}405.11728\left[\mathrm{M}-\mathrm{H}-\mathrm{C}_{7} \mathrm{H}_{4} \mathrm{O}_{4}\right]^{-} \\
243.06511\left[\mathrm{M}-\mathrm{H}-\mathrm{C}_{7} \mathrm{H}_{4} \mathrm{O}_{4}-\mathrm{C}_{6} \mathrm{H}_{10} \mathrm{O}_{5}\right]^{-} \\
225.05495\left[\mathrm{M}-\mathrm{H}-\mathrm{C}_{7} \mathrm{H}_{4} \mathrm{O}_{4}-\mathrm{C}_{6} \mathrm{H}_{10} \mathrm{O}_{5^{-}}\right. \\
\left.\mathrm{H}_{2} \mathrm{O}\right]^{-}\end{array}$ & $\begin{array}{l}\text { PMRPW, } \\
\text { PMRPE }\end{array}$ \\
\hline 73 & 10.70 & $\begin{array}{l}\text { Tetrahydroxystilbene-O- } \\
\text { (caffeoyl)-glucoside }{ }^{\text {a }}\end{array}$ & $\mathrm{C}_{29} \mathrm{H}_{28} \mathrm{O}_{12}$ & 567.14502 & 567.14970 & -8.256 & $243.06516\left[\mathrm{M}-\mathrm{H}-\mathrm{C}_{6} \mathrm{H}_{10} \mathrm{O}_{5}-\mathrm{C}_{9} \mathrm{H}_{6} \mathrm{O}_{3}\right]^{-}$ & PMRPE \\
\hline 75 & 10.81 & Polydatin ${ }^{a b}$ & $\mathrm{C}_{20} \mathrm{H}_{22} \mathrm{O}_{8}$ & 389.12457 & 389.12309 & 0.984 & $\begin{array}{l}227.07018\left[\mathrm{M}-\mathrm{H}-\mathrm{C}_{6} \mathrm{H}_{10} \mathrm{O}_{5}\right]^{-} \\
209.05940\left[\mathrm{M}-\mathrm{H}-\mathrm{C}_{6} \mathrm{H}_{10} \mathrm{O}_{5}-\mathrm{H}_{2} \mathrm{O}\right]^{-} \\
199.07462\left[\mathrm{M}-\mathrm{H}-\mathrm{C}_{6} \mathrm{H}_{10} \mathrm{O}_{5}-\mathrm{CO}\right]^{-}\end{array}$ & $\begin{array}{l}\text { PMRPW, } \\
\text { PMRPE }\end{array}$ \\
\hline 76 & 10.83 & 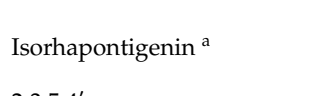 & $\mathrm{C}_{15} \mathrm{H}_{14} \mathrm{O}_{4}$ & 257.08127 & 257.08084 & 1.691 & $\begin{array}{l}242.05462\left[\mathrm{M}-\mathrm{H}-\mathrm{CH}_{3}\right]^{-} \\
187.56930\left[\mathrm{M}-\mathrm{H}-\mathrm{CH}_{2}-2 \mathrm{CO}\right]^{-} \\
136.18150\left[\mathrm{M}-\mathrm{H}-\mathrm{C}_{7} \mathrm{H}_{5} \mathrm{O}_{2}\right]^{-}\end{array}$ & $\begin{array}{l}\text { PMRPW, } \\
\text { PMRPE }\end{array}$ \\
\hline 81 & 11.39 & $\begin{array}{l}2,3,5,4^{\prime} \text { - } \\
\text { Tetrahydroxystilbene-2-O- } \beta \text { - } \\
\text { D-(2"-O-coumaroyl)- } \\
\text { glucoside }\end{array}$ & $\mathrm{C}_{29} \mathrm{H}_{28} \mathrm{O}_{11}$ & 551.15112 & 551.15479 & -3.668 & $\begin{array}{l}\left.\text { 389.10031[M-H- } \mathrm{C}_{6} \mathrm{H}_{10} \mathrm{O}_{5}\right]^{-} \\
225.05389\left[\mathrm{M}-\mathrm{H}-\mathrm{C}_{6} \mathrm{H}_{10} \mathrm{O}_{5}-\mathrm{C}_{9} \mathrm{H}_{6} \mathrm{O}_{2^{-}}\right. \\
\left.\mathrm{H}_{2} \mathrm{O}\right]^{-}\end{array}$ & PMRPE \\
\hline 88 & 13.17 & $\begin{array}{l}\text { Tetrahydroxystilbene-2- } \\
\text { (feruloyl)- } \\
\text { glucoside }\end{array}$ & $\mathrm{C}_{30} \mathrm{H}_{30} \mathrm{O}_{12}$ & 581.16583 & 581.16535 & 0.821 & $\begin{array}{l}\left.\text { 419.11136[M-H- } \mathrm{C}_{6} \mathrm{H}_{10} \mathrm{O}_{5}\right]^{-} \\
405.21970\left[\mathrm{M}-\mathrm{H}-\mathrm{C}_{10} \mathrm{H}_{8} \mathrm{O}_{3}\right]^{-} \\
295.05981\left[\mathrm{M}-\mathrm{H}-\mathrm{C}_{6} \mathrm{H}_{10} \mathrm{O}_{5}-\mathrm{C}_{6} \mathrm{H}_{4} \mathrm{O}_{3}\right]^{-}\end{array}$ & $\begin{array}{l}\text { PMRPW, } \\
\text { PMRPE }\end{array}$ \\
\hline \multicolumn{9}{|c|}{ Flavonoids and derivatives } \\
\hline 18 & 3.63 & $\begin{array}{l}\text { Liquiritigenin-glucoside- } \\
\text { xyl/ara }\end{array}$ & $\mathrm{C}_{26} \mathrm{H}_{30} \mathrm{O}_{13}$ & 549.1604 & 549.16027 & 0.023 & $\begin{array}{l}\left.\text { 387.10541[M-H- } \mathrm{C}_{6} \mathrm{H}_{10} \mathrm{O}_{5}\right]^{-} \\
369.09552\left[\mathrm{M}-\mathrm{H}-\mathrm{C}_{6} \mathrm{H}_{10} \mathrm{O}_{5}-\mathrm{H}_{2} \mathrm{O}\right]^{-} \\
279.06604\left[\mathrm{M}-\mathrm{H}-\mathrm{C}_{6} \mathrm{H}_{10} \mathrm{O}_{5}-\mathrm{C}_{5} \mathrm{H}_{8} \mathrm{O}_{4}\right]^{-} \\
151.03955\left[\mathrm{M}-\mathrm{H}-\mathrm{C}_{7} \mathrm{H}_{5} \mathrm{O}_{3}\right]^{-}\end{array}$ & $\begin{array}{l}\text { PMRPW, } \\
\text { PMRPE }\end{array}$ \\
\hline 28 & 4.79 & Catechin & $\mathrm{C}_{15} \mathrm{H}_{14} \mathrm{O}_{6}$ & 289.07111 & 289.07066 & 1.541 & $\begin{array}{l}137.02376\left[\mathrm{M}-\mathrm{H}-\mathrm{C}_{8} \mathrm{H}_{7} \mathrm{O}_{3}\right]^{-} \\
123.04458\left[\mathrm{M}-\mathrm{H}-\mathrm{C}_{7} \mathrm{H}_{5} \mathrm{O}_{3}-\mathrm{CO}\right]^{-} \\
109.02898\left[\mathrm{M}-\mathrm{H}-\mathrm{C}_{8} \mathrm{H}_{7} \mathrm{O}_{3}-\mathrm{C}_{2} \mathrm{H}_{2} \mathrm{O}\right]^{-} \\
151.03955\left[\mathrm{M}-\mathrm{H}-\mathrm{C}_{7} \mathrm{H}_{5} \mathrm{O}_{3}\right]^{-}\end{array}$ & $\begin{array}{l}\text { PMRPW, } \\
\text { PMRPE }\end{array}$ \\
\hline 31 & 5.93 & Epicatechin & $\mathrm{C}_{15} \mathrm{H}_{14} \mathrm{O}_{6}$ & 289.07080 & 289.07066 & 0.468 & $\begin{array}{l}137.02376\left[\mathrm{M}-\mathrm{H}-\mathrm{C}_{8} \mathrm{H}_{7} \mathrm{O}_{3}\right]^{-} \\
123.04458\left[\mathrm{M}-\mathrm{H}-\mathrm{C}_{7} \mathrm{H}_{5} \mathrm{O}_{3}-\mathrm{CO}\right]^{-} \\
109.02898\left[\mathrm{M}-\mathrm{H}-\mathrm{C}_{8} \mathrm{H}_{7} \mathrm{O}_{3}-\mathrm{C}_{2} \mathrm{H}_{2} \mathrm{O}\right]^{-} \\
330.07205\left[\mathrm{M}-\mathrm{H}-\mathrm{C}_{6} \mathrm{H}_{11} \mathrm{O}_{5}\right]^{-}\end{array}$ & $\begin{array}{l}\text { PMRPW, } \\
\text { PMRPE }\end{array}$ \\
\hline 35 & 6.36 & $\begin{array}{l}\text { Acetyl-epicatechin-O- } \\
\text { glucoside }^{\text {a }}\end{array}$ & $\mathrm{C}_{23} \mathrm{H}_{26} \mathrm{O}_{12}$ & 493.13406 & 493.13405 & 0.015 & $\begin{array}{l}255.06543\left[\mathrm{M}-\mathrm{H}-\mathrm{C}_{6} \mathrm{H}_{10} \mathrm{O}_{5}-\mathrm{C}_{2} \mathrm{H}_{2} \mathrm{O}_{2-}\right. \\
\left.\mathrm{H}_{2} \mathrm{O}\right]^{-} \\
227.07016\left[\mathrm{M}-\mathrm{H}-\mathrm{C}_{6} \mathrm{H}_{10} \mathrm{O}_{5}-\mathrm{C}_{2} \mathrm{H}_{2} \mathrm{O}_{2-}^{-}\right. \\
\left.\mathrm{H}_{2} \mathrm{O}-\mathrm{CO}\right]^{-}\end{array}$ & $\begin{array}{l}\text { PMRPW, } \\
\text { PMRPE }\end{array}$ \\
\hline 39 & 7.04 & Hesperetin-7-O-glucoside ${ }^{\text {a }}$ & $\mathrm{C}_{22} \mathrm{H}_{24} \mathrm{O}_{11}$ & 463.12482 & 463.12349 & 2.876 & $\begin{array}{l}\left.\text { 419.13446[M-H-CO } \mathrm{CO}_{2}\right]^{-} \\
256.07315\left[\mathrm{M}-\mathrm{H}-\mathrm{CO}_{2}-\mathrm{C}_{6} \mathrm{H}_{11} \mathrm{O}_{5}\right]^{-}\end{array}$ & $\begin{array}{l}\text { PMRPW, } \\
\text { PMRPE }\end{array}$ \\
\hline 49 & 7.73 & $\begin{array}{l}\text { Trihydroxy- } \\
\text { dimethoxychalcone-O- } \\
\text { glucoside }\end{array}$ & $\mathrm{C}_{23} \mathrm{H}_{26} \mathrm{O}_{11}$ & 477.13867 & 477.13914 & -0.981 & $\begin{array}{l}315.08606\left[\mathrm{M}-\mathrm{H}-\mathrm{C}_{6} \mathrm{H}_{10} \mathrm{O}_{5}\right]^{-} \\
297.07486\left[\mathrm{M}-\mathrm{H}-\mathrm{C}_{6} \mathrm{H}_{10} \mathrm{O}_{5}-\mathrm{H}_{2} \mathrm{O}\right]^{-} \\
243.06522\left[\mathrm{M}-\mathrm{H}-\mathrm{C}_{6} \mathrm{H}_{10} \mathrm{O}_{5}-2 \mathrm{CO}-\mathrm{O}\right]^{-} \\
289.07086\left[\mathrm{M}-\mathrm{H}-\mathrm{C}_{7} \mathrm{H}_{5} \mathrm{O}_{4}\right]^{-}\end{array}$ & $\begin{array}{l}\text { PMRPW, } \\
\text { PMRPE }\end{array}$ \\
\hline 51 & 7.90 & Epicatechin-O-gallate & $\mathrm{C}_{22} \mathrm{H}_{18} \mathrm{O}_{10}$ & 441.08109 & 441.08162 & -3.706 & $\begin{array}{l}243.06519\left[\mathrm{M}-\mathrm{H}-\mathrm{C}_{7} \mathrm{H}_{5} \mathrm{O}_{4}-\mathrm{H}_{2} \mathrm{O}-\right. \\
\mathrm{CO}]^{-} 225.05489\left[\mathrm{M}-\mathrm{H}_{-}-\mathrm{C}_{7} \mathrm{H}_{5} \mathrm{O}_{4}-\mathrm{CO}_{2}\right]^{-} \\
169.01367\left[\mathrm{M}-\mathrm{H}-\mathrm{C}_{15} \mathrm{H}_{13} \mathrm{O}_{5}\right]^{-} \\
313.06976\left[\mathrm{M}-\mathrm{H}-\mathrm{C}_{6} \mathrm{H}_{10} \mathrm{O}_{5}\right]^{-}\end{array}$ & PMRPE \\
\hline 55 & 8.19 & Cirsimarin ${ }^{a}$ & $\mathrm{C}_{23} \mathrm{H}_{24} \mathrm{O}_{11}$ & 475.12415 & 475.12349 & 1.394 & $\begin{array}{l}285.07596\left[\mathrm{M}-\mathrm{H}-\mathrm{C}_{6} \mathrm{H}_{10} \mathrm{O}_{5}-\mathrm{CO}\right]^{-} \\
242.05670\left[\mathrm{M}-\mathrm{H}-\mathrm{C}_{6} \mathrm{H}_{10} \mathrm{O}_{5}-\mathrm{CO}_{2-}^{-}\right. \\
\left.2 \mathrm{CH}_{2}\right]^{-}\end{array}$ & $\begin{array}{l}\text { PMRPW, } \\
\text { PMRPE }\end{array}$ \\
\hline 59 & 9.03 & Epimedium & $\mathrm{C}_{20} \mathrm{H}_{20} \mathrm{O}_{7}$ & 371.11102 & 371.11253 & -4.067 & $\begin{array}{l}281.08215\left[\mathrm{M}-\mathrm{H}-\mathrm{C}_{4} \mathrm{H}_{10} \mathrm{O}_{2}\right]^{-} \\
161.02383\left[\mathrm{M}-\mathrm{H}-\mathrm{C}_{4} \mathrm{H}_{8} \mathrm{O}_{2}-\mathrm{C}_{7} \mathrm{H}_{4} \mathrm{O}_{2}\right]^{-} \\
285.03925\left[\mathrm{M}-\mathrm{H}-\mathrm{C}_{6} \mathrm{H}_{10} \mathrm{O}_{5}\right]^{-}\end{array}$ & PMRPE \\
\hline 60 & 9.11 & Kaempferol-3- $\beta$-D-glucoside & $\mathrm{C}_{21} \mathrm{H}_{20} \mathrm{O}_{11}$ & 447.09103 & 447.09219 & -3.529 & $\begin{array}{l}285.03925\left[\mathrm{M}-\mathrm{H}-\mathrm{C}_{6} \mathrm{H}_{10} \mathrm{O}_{5}\right]^{-} \\
257.04456\left[\mathrm{M}-\mathrm{H}-\mathrm{C}_{6} \mathrm{H}_{10} \mathrm{O}_{5}-\mathrm{CO}\right]^{-} \\
229.04724\left[\mathrm{M}-\mathrm{H}-\mathrm{C}_{6} \mathrm{H}_{10} \mathrm{O}_{5}-\mathrm{CO}\right]^{-}\end{array}$ & $\begin{array}{l}\text { PMRPW, } \\
\text { PMRPE }\end{array}$ \\
\hline 63 & 9.49 & Quercetin & $\mathrm{C} 15 \mathrm{H} 10 \mathrm{O} 7$ & 301.03424 & 301.03428 & -0.130 & $\begin{array}{l}283.03264[\mathrm{M}-\mathrm{H}-\mathrm{H} 2 \mathrm{O}]^{-} \\
273.04050[\mathrm{M}-\mathrm{H}-\mathrm{CO}]^{-} \\
255.02896[\mathrm{M}-\mathrm{H}-\mathrm{CO}-\mathrm{H} 2 \mathrm{O}]^{-}\end{array}$ & $\begin{array}{l}\text { PMRPW, } \\
\text { PMRPE }\end{array}$ \\
\hline 65 & 9.55 & Kaempferol ${ }^{\text {a }}$ & $\mathrm{C}_{15} \mathrm{H}_{10} \mathrm{O}_{6}$ & 285.04022 & 285.03936 & 3.001 & $\begin{array}{l}241.04958\left[\mathrm{M}-\mathrm{H}-\mathrm{CO}_{2}\right]^{-} \\
257.67783[\mathrm{M}-\mathrm{H}-\mathrm{CO}]^{-}\end{array}$ & $\begin{array}{l}\text { PMRPW, } \\
\text { PMRPE }\end{array}$ \\
\hline 70 & 10.27 & $\begin{array}{l}\text { Kaempferol-O-glucoside- } \\
\text { rhamnose }^{\text {a }}\end{array}$ & $\mathrm{C}_{27} \mathrm{H}_{30} \mathrm{O}_{15}$ & 593.14893 & 593.15010 & -1.967 & $\begin{array}{l}269.04529\left[\mathrm{M}-\mathrm{H}-2 \mathrm{C}_{6} \mathrm{H}_{10} \mathrm{O}_{5}\right]^{-} \\
225.05469\left[\mathrm{M}-\mathrm{H}-2 \mathrm{C}_{6} \mathrm{H}_{10} \mathrm{O}_{5}-\mathrm{CO}_{2}\right]^{-} \\
241.04984\left[\mathrm{M}-\mathrm{H}-2 \mathrm{C}_{6} \mathrm{H}_{10} \mathrm{O}_{5}-\mathrm{CO}\right]^{-}\end{array}$ & $\begin{array}{l}\text { PMRPW, } \\
\text { PMRPE }\end{array}$ \\
\hline 74 & 10.76 & Dihydroquercetin & $\mathrm{C}_{15} \mathrm{H}_{12} \mathrm{O}_{7}$ & 303.04868 & 303.04929 & -2.109 & $\begin{array}{l}\left.\text { 151.03946[M-H-CO } \mathrm{CO}_{2}-\mathrm{C}_{6} \mathrm{H}_{4} \mathrm{O}_{2}\right]^{-} \\
153.01883\left[\mathrm{M}-\mathrm{H}-\mathrm{C}_{8} \mathrm{H}_{7} \mathrm{O}_{3}\right]^{-} \\
125.02396\left[\mathrm{M}-\mathrm{H}-\mathrm{C}_{8} \mathrm{H}_{7} \mathrm{O}_{3}-\mathrm{CO}\right]^{-}\end{array}$ & $\begin{array}{l}\text { PMRPW, } \\
\text { PMRPE }\end{array}$ \\
\hline
\end{tabular}


Table 1. Cont.

\begin{tabular}{|c|c|c|c|c|c|c|c|c|}
\hline NO. & $\begin{array}{l}\mathrm{RT} \\
(\mathrm{min})\end{array}$ & Identification & $\begin{array}{l}\text { Molecular } \\
\text { Formula }\end{array}$ & $\begin{array}{l}\text { Measured } \\
\text { Mass }[\mathrm{M}-\mathrm{H}]^{-}\end{array}$ & $\begin{array}{l}\text { Accuracy } \\
\text { Mass }[\mathbf{M}-\mathbf{H}]^{-}\end{array}$ & $\begin{array}{l}\text { Error } \\
\text { (ppm) }\end{array}$ & Characteristic Fragment Ions & Source \\
\hline \multicolumn{9}{|c|}{ Phenolic acids and derivatives } \\
\hline 9 & 1.99 & Gallic acid ${ }^{b}$ & $\mathrm{C}_{7} \mathrm{H}_{6} \mathrm{O}_{5}$ & 169.01358 & 169.01315 & 2.546 & $\begin{array}{l}\left.\text { 125.02383[M-H-CO } \mathrm{CO}_{2}\right]^{-} \\
107.01351\left[\mathrm{M}-\mathrm{H}-\mathrm{CO}_{2}-\mathrm{H}_{2} \mathrm{O}\right]^{-} \\
97.02921\left[\mathrm{M}-\mathrm{H}-\mathrm{CO}_{2}-\mathrm{CO}\right]^{-}\end{array}$ & $\begin{array}{l}\text { PMRPW, } \\
\text { PMRPE }\end{array}$ \\
\hline 10 & 2.06 & $\begin{array}{l}\text { Gallic acid-O- } \\
\text { glucoside }\end{array}$ & $\mathrm{C}_{13} \mathrm{H}_{16} \mathrm{O}_{10}$ & 331.06595 & 331.06597 & -0.070 & $\begin{array}{l}169.01357\left[\mathrm{M}-\mathrm{H}-\mathrm{C}_{6} \mathrm{H}_{10} \mathrm{O}_{5}\right]^{-} \\
125.02380\left[\mathrm{M}-\mathrm{H}-\mathrm{C}_{6} \mathrm{H}_{10} \mathrm{O}_{5}-\mathrm{CO}_{2}\right]^{-}\end{array}$ & $\begin{array}{l}\text { PMRPW, } \\
\text { PMRPE }\end{array}$ \\
\hline 12 & 2.75 & Dihydroxy-benzoic acid ${ }^{a}$ & $\mathrm{C}_{7} \mathrm{H}_{6} \mathrm{O}_{4}$ & 153.01859 & 153.01824 & 2.319 & $\begin{array}{l}125.02429[\mathrm{M}-\mathrm{H}-\mathrm{CO}]^{-} \\
109.02917\left[\mathrm{M}-\mathrm{H}-\mathrm{CO}_{2}\right]^{-}\end{array}$ & $\begin{array}{l}\text { PMRPW, } \\
\text { PMRPE }\end{array}$ \\
\hline 14 & 2.83 & Galloyl-glycerol ${ }^{\text {a }}$ & $\mathrm{C}_{10} \mathrm{H}_{12} \mathrm{O}_{7}$ & 243.04993 & 243.04993 & 0.004 & $\begin{array}{l}\left.\text { 169.01321[M-H- } \mathrm{C}_{3} \mathrm{H}_{6} \mathrm{O}_{2}\right]^{-} \\
125.02386\left[\mathrm{M}-\mathrm{H}-\mathrm{C}_{3} \mathrm{H}_{6} \mathrm{O}_{2}-\mathrm{CO}_{2}\right]^{-} \\
118.96574\left[\mathrm{M}-\mathrm{H}-\mathrm{C}_{3} \mathrm{H}_{6} \mathrm{O}_{2}-3 \mathrm{O}\right]^{-}\end{array}$ & $\begin{array}{l}\text { PMRPW, } \\
\text { PMRPE }\end{array}$ \\
\hline 15 & 2.90 & Vanillic acid ${ }^{a}$ & $\mathrm{C}_{8} \mathrm{H}_{8} \mathrm{O}_{4}$ & 167.03433 & 167.03389 & -0.331 & $\begin{array}{l}137.02341\left[\mathrm{M}-\mathrm{H}-\mathrm{CH}_{2} \mathrm{O}\right]^{-} \\
123.04459\left[\mathrm{M}-\mathrm{H}-\mathrm{CO}_{2}\right]^{-}\end{array}$ & $\begin{array}{l}\text { PMRPW, } \\
\text { PMRPE }\end{array}$ \\
\hline 16 & 3.30 & $\begin{array}{l}\text { Isomer Dihydroxy- } \\
\text { benzoic acid }\end{array}$ & $\mathrm{C}_{7} \mathrm{H}_{6} \mathrm{O}_{4}$ & 153.01859 & 153.01824 & -0.230 & $\begin{array}{l}137.45282[\mathrm{M}-\mathrm{H}-\mathrm{O}]^{-} \\
125.02434[\mathrm{M}-\mathrm{H}-\mathrm{CO}]^{-} \\
109.02898\left[\mathrm{M}-\mathrm{H}-\mathrm{CO}_{2}\right]^{-}\end{array}$ & $\begin{array}{l}\text { PMRPW, } \\
\text { PMRPE }\end{array}$ \\
\hline 17 & 3.34 & $\begin{array}{l}\text { Protocatechuic } \\
\text { acid-O-glucoside }\end{array}$ & $\mathrm{C}_{13} \mathrm{H}_{16} \mathrm{O}_{9}$ & 315.06995 & 315.07106 & -3.518 & $\begin{array}{l}\left.\text { 153.01865[M-H- }{ }_{6} \mathrm{H}_{10} \mathrm{O}_{5}\right]^{-} \\
109.02901\left[\mathrm{M}-\mathrm{H}-\mathrm{C}_{6} \mathrm{H}_{10} \mathrm{O}_{5}-\mathrm{CO}_{2}\right]^{-}\end{array}$ & $\begin{array}{l}\text { PMRPW, } \\
\text { PMRPE }\end{array}$ \\
\hline 19 & 3.71 & Caffeic acid ${ }^{\text {a }}$ & $\mathrm{C}_{9} \mathrm{H}_{8} \mathrm{O}_{4}$ & 179.03412 & 179.03389 & 2.093 & $\begin{array}{l}\left.\text { 135.04448[M-H-CO } \mathrm{CO}_{2}\right]^{-} \\
107.04977\left[\mathrm{M}-\mathrm{H}-\mathrm{C}_{3} \mathrm{H}_{4} \mathrm{O}_{2}\right]^{-}\end{array}$ & $\begin{array}{l}\text { PMRPW, } \\
\text { PMRPE }\end{array}$ \\
\hline 20 & 3.75 & $\begin{array}{l}\text { 1-(5-Methylfuran-2-yl) } \\
\text { ethanone }^{\text {a }}\end{array}$ & $\mathrm{C}_{7} \mathrm{H}_{8} \mathrm{O}_{2}$ & 123.04462 & 123.04406 & 4.584 & $\begin{array}{l}\text { 108.02116[M-H-CH}]_{3}^{-} \\
95.01338\left[\mathrm{M}-\mathrm{H}-\mathrm{CO}^{-}\right. \\
79.05503\left[\mathrm{M}-\mathrm{H}-\mathrm{CO}_{2}\right]^{-}\end{array}$ & $\begin{array}{l}\text { PMRPW, } \\
\text { PMRPE }\end{array}$ \\
\hline 21 & 4.16 & Veratric acid ${ }^{a}$ & $\mathrm{C}_{9} \mathrm{H}_{10} \mathrm{O}_{4}$ & 181.05014 & 181.04954 & 2.567 & $\begin{array}{l}\left.\text { 137.02396[M-H-CO }{ }_{2}\right]^{-} \\
122.03658\left[\mathrm{M}-\mathrm{H}-2 \mathrm{CH}_{2} \mathrm{O}\right]^{-} \\
107.04949\left[\mathrm{M}-\mathrm{H}-\mathrm{CO}-\mathrm{CH}_{2} \mathrm{O}\right]^{-}\end{array}$ & $\begin{array}{l}\text { PMRPW, } \\
\text { PMRPE }\end{array}$ \\
\hline 25 & 4.56 & 3-Hydroxybenzoic acid & $\mathrm{C}_{7} \mathrm{H}_{6} \mathrm{O}_{3}$ & 137.02371 & 137.02332 & 2.842 & $\begin{array}{l}119.01297\left[\mathrm{M}-\mathrm{H}-\mathrm{H}_{2} \mathrm{O}\right]^{-} \\
93.03405\left[\mathrm{M}-\mathrm{H}-\mathrm{CO}_{2}\right]^{-}\end{array}$ & $\begin{array}{l}\text { PMRPW, } \\
\text { PMRPE }\end{array}$ \\
\hline 26 & 4.75 & Coumaric acid ${ }^{a}$ & $\mathrm{C}_{9} \mathrm{H}_{8} \mathrm{O}_{3}$ & 163.03937 & 163.03897 & 2.4500 & $\begin{array}{l}\left.\text { 119.04978[M-H-CO }{ }_{2}\right]^{-} \\
134.91408\left[\mathrm{M}-\mathrm{H}-\mathrm{CO}^{-}\right. \\
107.04973\left[\mathrm{M}-\mathrm{H}-\mathrm{C}_{2} \mathrm{O}_{2}\right]^{-}\end{array}$ & $\begin{array}{l}\text { PMRPW, } \\
\text { PMRPE }\end{array}$ \\
\hline 27 & 4.77 & 2-Methyl gallic acid a & $\mathrm{C}_{8} \mathrm{H}_{8} \mathrm{O}_{5}$ & 183.02893 & 183.02880 & 0.711 & $\begin{array}{l}\text { 168.00560[M-H-CH}]_{3}^{-} \\
139.00296\left[\mathrm{M}-\mathrm{H}-\mathrm{CO}_{2}\right]^{-} \\
111.00824\left[\mathrm{M}-\mathrm{H}-\mathrm{CO}_{2}-\mathrm{CO}\right]^{-}\end{array}$ & $\begin{array}{l}\text { PMRPW, } \\
\text { PMRPE }\end{array}$ \\
\hline 34 & 6.24 & Methyl gallate ${ }^{a}$ & $\mathrm{C}_{8} \mathrm{H}_{8} \mathrm{O}_{4}$ & 167.03424 & 167.03389 & 2.124 & $\begin{array}{l}151.00310\left[\mathrm{M}-\mathrm{H}-\mathrm{CH}_{3}\right]^{-} \\
125.02368\left[\mathrm{M}-\mathrm{H}-\mathrm{C}_{2} \mathrm{H}_{2} \mathrm{O}\right]^{-} 107.01314[\mathrm{M}- \\
\left.\mathrm{H}-\mathrm{C}_{2} \mathrm{H}_{2} \mathrm{O}_{2}\right]^{-}\end{array}$ & $\begin{array}{l}\text { PMRPW, } \\
\text { PMRPE }\end{array}$ \\
\hline 40 & 7.06 & Syringic acid ${ }^{a}$ & $\mathrm{C}_{9} \mathrm{H}_{10} \mathrm{O}_{5}$ & 197.04417 & 197.04445 & -1.420 & $\begin{array}{l}169.01370[\mathrm{M}-\mathrm{H}-\mathrm{CO}]^{-} \\
125.02389\left[\mathrm{M}-\mathrm{H}-\mathrm{CO}-\mathrm{CO}_{2}\right]^{-}\end{array}$ & PMRPE \\
\hline \multicolumn{9}{|c|}{ Others } \\
\hline 1 & 0.73 & L-Arginine & $\mathrm{C}_{6} \mathrm{H}_{14} \mathrm{~N}_{4} \mathrm{O}_{2}$ & 173.10316 & 173.10330 & -0.821 & $\begin{array}{l}\left.\text { 131.08197[M-H-CN } \mathrm{CH}_{2}\right]^{-} \\
114.05562\left[\mathrm{M}-\mathrm{H}-\mathrm{NH}-\mathrm{CO}_{2}\right]^{-} \\
161.06087\left[\mathrm{M}-\mathrm{H}-\mathrm{H}_{2} \mathrm{O}\right]^{-}\end{array}$ & $\begin{array}{l}\text { PMRPW, } \\
\text { PMRPE }\end{array}$ \\
\hline 2 & 0.79 & Glucose & $\mathrm{C}_{6} \mathrm{H}_{12} \mathrm{O}_{6}$ & 179.05534 & 179.05501 & 1.818 & $\begin{array}{l}\left.\text { 131.03432[M-H- } \mathrm{H}_{2} \mathrm{O}-\mathrm{CH}_{2} \mathrm{O}\right]^{-} \\
85.02903\left[\mathrm{M}-\mathrm{H}-\mathrm{CH}_{2} \mathrm{O}-4 \mathrm{O}\right]^{-}\end{array}$ & $\begin{array}{l}\text { PMRPW, } \\
\text { PMRPE }\end{array}$ \\
\hline 3 & 0.82 & L-Threonine & $\mathrm{C}_{4} \mathrm{H}_{9} \mathrm{NO}_{3}$ & 118.05041 & 118.04987 & 4.577 & $\begin{array}{l}74.02446\left[\mathrm{M}-\mathrm{H}-\mathrm{CO}_{2}\right]^{-} \\
59.01369\left[\mathrm{M}-\mathrm{H}-\mathrm{CO}_{2}-\mathrm{CH}_{3}\right]^{-}\end{array}$ & $\begin{array}{l}\text { PMRPW, } \\
\text { PMRPE }\end{array}$ \\
\hline 4 & 0.85 & $\begin{array}{l}\text { (2S)-2-Hydroxybutanedioic } \\
\text { acid }\end{array}$ & $\mathrm{C}_{4} \mathrm{H}_{6} \mathrm{O}_{5}$ & 133.01361 & 133.01315 & 3.460 & $\begin{array}{l}\left.\text { 115.00312[M-H-H } \mathrm{H}_{2} \mathrm{O}\right]^{-} \\
89.02412\left[\mathrm{M}-\mathrm{H}-\mathrm{CO}_{2}\right]^{-} \\
71.01358\left[\mathrm{M}-\mathrm{H}-\mathrm{H}_{2} \mathrm{O}-\mathrm{CO}_{2}\right]^{-}\end{array}$ & $\begin{array}{l}\text { PMRPW, } \\
\text { PMRPE }\end{array}$ \\
\hline 5 & 1.29 & Citric acid ${ }^{a}$ & $\mathrm{C}_{6} \mathrm{H}_{8} \mathrm{O}_{7}$ & 191.01889 & 191.01863 & 1.366 & $\begin{array}{l}129.01921\left[\mathrm{M}-\mathrm{H}-\mathrm{CO}_{2}-\mathrm{H}_{2} \mathrm{O}\right]^{-} \\
111.00829\left[\mathrm{M}-\mathrm{H}-\mathrm{CO}_{2}-2 \mathrm{H}_{2} \mathrm{O}\right]^{-} \\
87.00838\left[\mathrm{M}-\mathrm{H}-2 \mathrm{CO}_{2}-\mathrm{O}\right]^{-}\end{array}$ & $\begin{array}{l}\text { PMRPW, } \\
\text { PMRPE }\end{array}$ \\
\hline 6 & 1.39 & L-Tyrosine $^{a}$ & $\mathrm{C}_{9} \mathrm{H}_{11} \mathrm{NO}_{3}$ & 180.06575 & 180.06552 & 1.279 & $\begin{array}{l}\text { 163.03926[M-H-OH }]^{-} \\
137.02368[\mathrm{M}-\mathrm{H}-\mathrm{NH}-\mathrm{CO}]^{-} \\
119.04951\left[\mathrm{M}-\mathrm{H}-\mathrm{CO}_{2}-\mathrm{OH}\right]^{-}\end{array}$ & $\begin{array}{l}\text { PMRPW, } \\
\text { PMRPE }\end{array}$ \\
\hline 7 & 1.40 & 3-O-feruloylquinic acid ${ }^{a}$ & $\mathrm{C}_{17} \mathrm{H}_{20} \mathrm{O}_{9}$ & 367.10272 & 367.10236 & 0.985 & $\begin{array}{l}\left.\text { 277.07294[M-H-COOH-CH}-\mathrm{CH}_{2} \mathrm{O}-\mathrm{H}_{2} \mathrm{O}\right]^{-} \\
157.03020\left[\mathrm{M}-\mathrm{H}-\mathrm{C}_{10} \mathrm{H}_{8} \mathrm{O}_{3}-2 \mathrm{OH}\right]^{-}\end{array}$ & $\begin{array}{l}\text { PMRPW, } \\
\text { PMRPE }\end{array}$ \\
\hline 8 & 1.43 & Leucine & $\mathrm{C}_{6} \mathrm{H}_{13} \mathrm{NO}_{2}$ & 130.08676 & 130.08626 & 3.881 & $\begin{array}{l}85.02912[\mathrm{M}-\mathrm{H}-\mathrm{COOH}]^{-} \\
88.04015\left[\mathrm{M}-\mathrm{H}-3 \mathrm{CH}_{2}\right]^{-}\end{array}$ & $\begin{array}{l}\text { PMRPW, } \\
\text { PMRPE }\end{array}$ \\
\hline 11 & 2.50 & $\begin{array}{l}\text { 3,5-Dihydroxy-2- } \\
\text { methyl-4hydro-pyran-4-one }\end{array}$ & $\mathrm{C}_{6} \mathrm{H}_{6} \mathrm{O}_{4}$ & 141.01833 & 141.01824 & 0.673 & $\begin{array}{l}\text { 112.95596[M-H-CO }]^{-} \\
97.02898\left[\mathrm{M}-\mathrm{H}-\mathrm{CO}_{2}\right]^{-} \\
69.03445\left[\mathrm{M}-\mathrm{H}-\mathrm{CO}_{2}-\mathrm{H}_{2} \mathrm{O}\right]^{-}\end{array}$ & $\begin{array}{l}\text { PMRPW, } \\
\text { PMRPE }\end{array}$ \\
\hline 13 & 2.79 & 5-Hydroxymethylfurfural & $\mathrm{C}_{6} \mathrm{H}_{6} \mathrm{O}_{3}$ & 125.02386 & 125.02332 & 2.795 & $\begin{array}{l}97.02918[\mathrm{M}-\mathrm{H}-\mathrm{CO}]^{-} \\
81.03435\left[\mathrm{M}-\mathrm{H}-\mathrm{CO}_{2}\right]^{-}\end{array}$ & $\begin{array}{l}\text { PMRPW, } \\
\text { PMRPE }\end{array}$ \\
\hline 22 & 4.35 & Altechromone $\mathrm{A}^{\mathrm{a}}$ & $\mathrm{C}_{11} \mathrm{H}_{10} \mathrm{O}_{3}$ & 189.05476 & 189.05462 & 0.737 & $\begin{array}{l}\text { 174.03186[M-H-CH }]_{3}^{-} \\
161.06018[\mathrm{M}-\mathrm{H}-\mathrm{CO}]^{-} \\
146.03635\left[\mathrm{M}-\mathrm{H}-\mathrm{CO}-\mathrm{CH}_{3}\right]^{-}\end{array}$ & $\begin{array}{l}\text { PMRPW, } \\
\text { PMRPE }\end{array}$ \\
\hline 23 & 4.52 & $\begin{array}{l}\text { Acetyl 1-methyl-1- } \\
\text { acetoxyethyl peroxide a }^{\text {a }}\end{array}$ & $\mathrm{C}_{7} \mathrm{H}_{12} \mathrm{O}_{5}$ & 175.06026 & 175.06010 & 0.914 & $\begin{array}{l}\left.\text { 160.97757[M-H-CH}]_{2}\right]^{-} \\
146.96054\left[\mathrm{M}-\mathrm{H}-2 \mathrm{CH}_{2}\right]^{-} \\
115.03953\left[\mathrm{M}-\mathrm{H}-2 \mathrm{CH}_{2} \mathrm{O}\right]^{-}\end{array}$ & $\begin{array}{l}\text { PMRPW, } \\
\text { PMRPE }\end{array}$ \\
\hline 24 & 4.56 & $\begin{array}{l}\text { 2-Vinyl-1H-indole-3- } \\
\text { carboxylic } \\
\text { acid }\end{array}$ & $\mathrm{C}_{11} \mathrm{H}_{9} \mathrm{NO}_{2}$ & 186.05539 & 186.05496 & 2.338 & $\begin{array}{l}\left.\text { 142.06551[M-H-CO } \mathrm{CO}_{2}\right]^{-} \\
159.93617\left[\mathrm{M}-\mathrm{H}-\mathrm{C}_{2} \mathrm{H}_{3}\right]^{-} \\
116.05013\left[\mathrm{M}-\mathrm{H}-\mathrm{C}_{2} \mathrm{H}_{2}-\mathrm{CO}_{2}\right]^{-}\end{array}$ & PMRPE \\
\hline 32 & 6.01 & P-hydroxybenzal-dehyde a & $\mathrm{C}_{7} \mathrm{H}_{6} \mathrm{O}_{2}$ & 121.0289 & 121.02841 & 4.082 & $93.03405[\mathrm{M}-\mathrm{H}-\mathrm{CO}]^{-}$ & $\begin{array}{l}\text { PMRPW, } \\
\text { PMRPE }\end{array}$ \\
\hline 36 & 6.54 & Vanillin $^{a}$ & $\mathrm{C}_{8} \mathrm{H}_{8} \mathrm{O}_{3}$ & 151.03902 & 151.03897 & 0.327 & $\begin{array}{l}\text { 136.01607[M-H-CH}]_{3}^{-} \\
123.04456\left[\mathrm{M}-\mathrm{H}-\mathrm{CO}^{-}\right. \\
107.04993\left[\mathrm{M}-\mathrm{H}-\mathrm{CO}_{2}\right]^{-}\end{array}$ & $\begin{array}{l}\text { PMRPW, } \\
\text { PMRPE }\end{array}$ \\
\hline
\end{tabular}


Table 1. Cont.

\begin{tabular}{|c|c|c|c|c|c|c|c|c|}
\hline NO. & $\begin{array}{l}\mathrm{RT} \\
(\mathrm{min})\end{array}$ & Identification & $\begin{array}{l}\text { Molecular } \\
\text { Formula }\end{array}$ & $\begin{array}{l}\text { Measured } \\
\text { Mass }[\mathbf{M}-\mathbf{H}]^{-}\end{array}$ & $\begin{array}{l}\text { Accuracy } \\
\text { Mass }[\mathbf{M}-\mathbf{H}]^{-}\end{array}$ & $\begin{array}{l}\text { Error } \\
\text { (ppm) }\end{array}$ & Characteristic Fragment Ions & Source \\
\hline 38 & 7.03 & $\begin{array}{l}\text { 6-Methoxyl-2-Acetyl-3- } \\
\text { methyljuglone-8-O- } \beta \text {-D- } \\
\text { glucoside }\end{array}$ & $\mathrm{C} 20 \mathrm{H} 22 \mathrm{O} 10$ & 421.11276 & 421.11292 & -0.388 & $\begin{array}{l}\text { 259.06027[M-H-C6H10O5] }{ }^{-} \\
241.04961[\mathrm{M}-\mathrm{H}-\mathrm{C} 6 \mathrm{H} 10 \mathrm{O} 5-\mathrm{H} 2 \mathrm{O}]^{-} \\
213.05441[\mathrm{M}-\mathrm{H}-\mathrm{C} 6 \mathrm{H} 10 \mathrm{O} 5-\mathrm{H} 2 \mathrm{O}-\mathrm{CO}]^{-} \\
389.15720\left[\mathrm{M}-\mathrm{H}-\mathrm{C}_{6} \mathrm{H}_{10} \mathrm{O}_{5}\right]^{-}\end{array}$ & $\begin{array}{l}\text { PMRPW, } \\
\text { PMRPE }\end{array}$ \\
\hline 42 & 7.13 & Nudiposide $^{a}$ & $\mathrm{C}_{27} \mathrm{H}_{36} \mathrm{O}_{12}$ & 551.21313 & 551.21230 & 1.501 & $\begin{array}{l}359.11261\left[\mathrm{M}-\mathrm{H}-\mathrm{C}_{6} \mathrm{H}_{10} \mathrm{O}_{5}-2 \mathrm{CH}_{2} \mathrm{O}\right]^{-} \\
341.09985\left[\mathrm{M}-\mathrm{H}-\mathrm{C}_{6} \mathrm{H}_{10} \mathrm{O}_{5}-2 \mathrm{CH}_{2} \mathrm{O}-\right. \\
\left.\mathrm{H}_{2} \mathrm{O}\right]^{-}\end{array}$ & $\begin{array}{l}\text { PMRPW, } \\
\text { PMRPE }\end{array}$ \\
\hline 43 & 7.21 & $\begin{array}{l}\text { (+)-lyoniresinol-2 } \alpha-O-\beta- \\
\text { glucoside }^{\text {a }}\end{array}$ & $\mathrm{C}_{28} \mathrm{H}_{38} \mathrm{O}_{13}$ & 581.22284 & 581.22287 & -0.994 & $\begin{array}{l}419.16949[\mathrm{M}-\mathrm{H}- \\
\left.\mathrm{C}_{6} \mathrm{H}_{10} \mathrm{O}_{5}\right]^{-} 389.12219\left[\mathrm{M}-\mathrm{H}_{-} \mathrm{C}_{6} \mathrm{H}_{10} \mathrm{O}_{5-}^{-}\right. \\
\left.\mathrm{CH}_{2} \mathrm{O}\right]^{-} 359.11096\left[\mathrm{M}-\mathrm{H}-\mathrm{C}_{6} \mathrm{H}_{10} \mathrm{O}_{5^{-}}\right. \\
\left.2 \mathrm{CH}_{2} \mathrm{O}\right]^{-}\end{array}$ & $\begin{array}{l}\text { PMRPW, } \\
\text { PMRPE }\end{array}$ \\
\hline 46 & 7.40 & $\begin{array}{l}\text { Isomer } \\
\text { Altechromone A }\end{array}$ & $\mathrm{C}_{11} \mathrm{H}_{10} \mathrm{O}_{3}$ & 189.0547 & 189.05462 & 0.420 & $\begin{array}{l}174.03166\left[\mathrm{M}-\mathrm{H}-\mathrm{CH}_{3}\right]^{-} \\
161.06047[\mathrm{M}-\mathrm{H}-\mathrm{CO}]^{-} \\
147.04448\left[\mathrm{M}-\mathrm{H}-\mathrm{CO}-\mathrm{CH}_{2}\right]^{-}\end{array}$ & $\begin{array}{l}\text { PMRPW, } \\
\text { PMRPE }\end{array}$ \\
\hline 53 & 8.08 & $\begin{array}{l}\text { Cinnamyl-galloyl-O- } \\
\text { glucoside }^{\mathrm{a}}\end{array}$ & $\mathrm{C}_{22} \mathrm{H}_{22} \mathrm{O}_{11}$ & 461.10611 & 461.10784 & -3.747 & $\begin{array}{l}\left.\text { 417.11594[M-H- } \mathrm{CO}_{2}\right]^{-} \\
254.05766\left[\mathrm{M}-\mathrm{H}-\mathrm{CO}_{2}-\mathrm{C}_{6} \mathrm{H}_{11} \mathrm{O}_{5}\right]^{-}\end{array}$ & $\begin{array}{l}\text { PMRPW, } \\
\text { PMRPE }\end{array}$ \\
\hline 54 & 8.11 & $\begin{array}{l}\text { 2-Methyl-5-carboxymethyl-7- } \\
\text { hydroxychromone a }^{\text {a }}\end{array}$ & $\mathrm{C}_{12} \mathrm{H}_{10} \mathrm{O}_{5}$ & 233.04443 & 233.04555 & -0.085 & $\begin{array}{l}205.04994[\mathrm{M}-\mathrm{H}-\mathrm{CO}]^{-} \\
191.03783\left[\mathrm{M}-\mathrm{H}-\mathrm{CO}-\mathrm{CH}_{2}\right]^{-} \\
161.02485\left[\mathrm{M}-\mathrm{H}-\mathrm{CO}-\mathrm{CO}_{2}\right]^{-}\end{array}$ & PMRPE \\
\hline 62 & 9.24 & Trans-N-caffeoyltyramine ${ }^{a}$ & $\mathrm{C}_{17} \mathrm{H}_{17} \mathrm{NO}_{4}$ & 298.10773 & 298.10738 & 1.159 & $\begin{array}{l}135.04459\left[\mathrm{M}-\mathrm{H}-\mathrm{C}_{9} \mathrm{H}_{7} \mathrm{O}_{3}\right]^{-} \\
178.04970\left[\mathrm{M}-\mathrm{H}-\mathrm{C}_{8} \mathrm{H}_{8} \mathrm{O}\right]^{-} \\
148.05200\left[\mathrm{M}-\mathrm{H}-\mathrm{C}_{8} \mathrm{H}_{6} \mathrm{O}-\mathrm{OH}\right]^{-}\end{array}$ & $\begin{array}{l}\text { PMRPW, } \\
\text { PMRPE }\end{array}$ \\
\hline 71 & 10.30 & Noreugenin $^{\mathrm{a}}$ & $\mathrm{C}_{10} \mathrm{H}_{8} \mathrm{O}_{4}$ & 191.03399 & 191.03389 & 0.549 & $\begin{array}{l}\left.\text { 149.02406[M-H-CO-CH}]_{2}\right]^{-} \\
147.04459\left[\mathrm{M}-\mathrm{H}-\mathrm{CO}_{2}\right]^{-}\end{array}$ & $\begin{array}{l}\text { PMRPW, } \\
\text { PMRPE }\end{array}$ \\
\hline 72 & 10.61 & $\begin{array}{l}\text { 1,2-Dihydroxypropane-1-(4- } \\
\text { hydroxy-phenyl) }^{\mathrm{a}}\end{array}$ & $\mathrm{C}_{9} \mathrm{H}_{12} \mathrm{O}_{3}$ & 167.07063 & 167.07053 & 1.552 & $\begin{array}{l}152.04729\left[\mathrm{M}-\mathrm{H}-\mathrm{CH}_{3}\right]^{-} \\
138.92862[\mathrm{M}-\mathrm{H}-\mathrm{CO}]^{-}\end{array}$ & $\begin{array}{l}\text { PMRPW, } \\
\text { PMRPE }\end{array}$ \\
\hline 79 & 10.98 & N-trans-Feruloyl tyramine & $\mathrm{C}_{18} \mathrm{H}_{19} \mathrm{NO}_{4}$ & 312.12286 & 312.12303 & -0.559 & $\begin{array}{l}\left.\text { 190.05000[M-H-C } \mathrm{C}_{7} \mathrm{H}_{6} \mathrm{O}_{2}\right]^{-} \\
178.05019\left[\mathrm{M}-\mathrm{H}-\mathrm{C}_{8} \mathrm{H}_{6} \mathrm{O}_{2}\right]^{-} \\
148.05235\left[\mathrm{M}-\mathrm{H}-\mathrm{C}_{9} \mathrm{H}_{10} \mathrm{NO}_{2}\right]^{-}\end{array}$ & $\begin{array}{l}\text { PMRPW, } \\
\text { PMRPE }\end{array}$ \\
\hline 80 & 11.34 & $\begin{array}{l}\text { Trans-N-Feruloyl-3-O- } \\
\text { methyldopamine }\end{array}$ & $\mathrm{C}_{19} \mathrm{H}_{21} \mathrm{NO}_{5}$ & 342.13211 & 342.13360 & -4.353 & $\begin{array}{l}327.10962\left[\mathrm{M}-\mathrm{H}-\mathrm{CH}_{3}\right]^{-} \\
178.05003\left[\mathrm{M}-\mathrm{H}-\mathrm{C}_{9} \mathrm{H}_{8} \mathrm{O}_{3}\right]^{-}\end{array}$ & $\begin{array}{l}\text { PMRPW, } \\
\text { PMRPE }\end{array}$ \\
\hline 82 & 11.43 & $\begin{array}{l}\text { Thunberginol C-6- } \\
O-\beta \text {-D-glucoside }{ }^{\text {a }}\end{array}$ & $\mathrm{C} 21 \mathrm{H} 22 \mathrm{O} 10$ & 433.11395 & 433.11292 & 2.371 & $\begin{array}{l}\text { 271.06082[M-H-C6H10O5 }]^{-} \\
253.05016[\mathrm{M}-\mathrm{H}-\mathrm{C} 6 \mathrm{H} 10 \mathrm{O} 5-\mathrm{H} 2 \mathrm{O}]^{-} \\
243.06531[\mathrm{M}-\mathrm{H}-\mathrm{C} 6 \mathrm{H} 10 \mathrm{O} 5-\mathrm{CO}]^{-} \\
230.05690\left[\mathrm{M}-\mathrm{H}-\mathrm{CH}_{3}\right]^{-}\end{array}$ & $\begin{array}{l}\text { PMRPW, } \\
\text { PMRPE }\end{array}$ \\
\hline 83 & 11.87 & Torachrysone $^{\mathrm{a}}$ & $\mathrm{C}_{14} \mathrm{H}_{14} \mathrm{O}_{4}$ & 245.08078 & 245.08084 & -0.226 & $\begin{array}{l}215.03368\left[\mathrm{M}-\mathrm{H}-\mathrm{CH}_{2} \mathrm{O}\right]^{-} \\
159.04398\left[\mathrm{M}-\mathrm{H}-\mathrm{CH}_{2} \mathrm{O}-\mathrm{CH}_{2}-\mathrm{C}_{2} \mathrm{H}_{2} \mathrm{O}\right]^{-}\end{array}$ & PMRPE \\
\hline 97 & 16.62 & $\begin{array}{l}\text { 3,8-Dihydroxy-1- } \\
\text { methoxyxanthone a }^{\text {a }}\end{array}$ & C14H10O5 & 257.04535 & 257.04445 & 3.502 & $\begin{array}{l}239.03391[\mathrm{M}-\mathrm{H}-\mathrm{H} 2 \mathrm{O}]^{-} \\
229.04854[\mathrm{M}-\mathrm{H}-\mathrm{CO}]^{-} \\
211.03917[\mathrm{M}-\mathrm{H}-\mathrm{CO}-\mathrm{H} 2 \mathrm{O}]^{-}\end{array}$ & $\begin{array}{l}\text { PMRPW, } \\
\text { PMRPE }\end{array}$ \\
\hline
\end{tabular}

Note: PMRPE: Ethanol extract of Polygoni Multiflori Radix Praeparata; PMRPW: Water extract of Polygoni Multiflori Radix Praeparata;

a means first reported in PMRP; ${ }^{b}$ means components compared with standards.

\subsubsection{Identification of Anthraquinones and Derivatives}

Anthraquinones, which have the pharmacological effects of being anti-inflammatory, anti-virus, anti-cancer, lipid-lowering, and anti-diabetes [26], are the primary compounds in PMRP. There has been much literature which has revealed that anthraquinones can attenuate liver damage and demonstrate an anti-cirrhosis effect by reducing lipid peroxidation and inhibiting the proliferation of hepatic stellate cells [27-29]. Moreover, emodin and its oxidative metabolites were deemed as the main xenobiotic components, as they can combine with glutathione (GSH) to disturb cellular GSH and fatty acid metabolism in the liver [30,31]. Most anthraquinones in this family produced the characteristic fragment ions at $m / z 269$ and $m / z 240$, and the loss of two CO sequentially could be considered as the characteristic fragment behavior of anthraquinones and their derivates. In detail, peak 84, with a retention time of $11.97 \mathrm{~min}$, generated an $[\mathrm{M}-\mathrm{H}]^{-}$ion with mass accuracy at $m / z$ 473.10583. The molecular formula was predicted as $\mathrm{C}_{23} \mathrm{H}_{22} \mathrm{O}_{11}$ using Xcalibur (Thermo Fisher Scientific) within 5 ppm. As shown in Figure 3, the characteristic fragment ions at $m / z 311.05438$ indicated a loss of glucuronic acid from the precursor ion at $m / z$ 473.10583. Characteristic ions at $m / z 269.04575$ and 282.05304, which could be identified as losing $\mathrm{C}_{2} \mathrm{H}_{2} \mathrm{O}$ and $\mathrm{CO}$ from $\mathrm{m} / z$ 311.05438, respectively, were obtained. The $[\mathrm{M}-\mathrm{H}]^{-}$ion fragmented into other characteristic ions at $\mathrm{m} / \mathrm{z} 254.05710, \mathrm{~m} / \mathrm{z} 240.04149$, and $m / z$ 225.05450, which corresponded to [M-H-glc- $\left.\mathrm{C}_{2} \mathrm{H}_{2} \mathrm{O}-\mathrm{CH}_{2}\right]^{-}$, [M-H-glc- $\left.\mathrm{C}_{2} \mathrm{H}_{3} \mathrm{O}-\mathrm{CO}\right]^{-}$ and $\left[\mathrm{M}-\mathrm{H} \text {-glc- } \mathrm{C}_{2} \mathrm{H}_{3} \mathrm{O}-\mathrm{CO}-\mathrm{CH}_{3}\right]^{-}$. It was putatively identified as 2 -acetylemodin-8-O- $\beta$-Dglucoside, and the proposed fragmentation pathways of 2-acetylemodin-8-O- $\beta$-D-glucoside are depicted in Figure 4. Peak $\mathbf{9 0}$ was found at $13.65 \mathrm{~min}$ and showed a precise molecular weight at $m / z$ 283.06113. The fragment ion at $m / z 268.03760$ was produced by losing $\mathrm{CH}_{3}$. 
Other characteristic ions at $m / z 240.04179$ and 212.04668 were observed by losing two CO successively. According to the in-house database and reference standard, compound 90 was identified as physcion. Peak 93 was found at 14.69 min and generated a $[\mathrm{M}-\mathrm{H}]^{-}$ ion at $m / z$ 299.05493. MS/MS fragment at $m / z 268.03696,253.04982$, and 240.04204 have corresponded to $\left[\mathrm{M}-\mathrm{H}-\mathrm{CH}_{3} \mathrm{O}\right]^{-}$, $\left[\mathrm{M}-\mathrm{H}-\mathrm{CH}_{3} \mathrm{O}-\mathrm{CH}_{3}\right]^{-}$and $\left[\mathrm{M}-\mathrm{H}-\mathrm{CH}_{3} \mathrm{O}-\mathrm{CO}\right]^{-}$. As a result, the compound was putatively identified as questinol. Peak 103 produced $[\mathrm{M}-\mathrm{H}]^{-}$ions at $m / z 269.04514$, and further characteristic fragment ions were acquired at $m / z 241.04941$ and 213.05467 by losing two CO successively. By comparing with the reference standard, the compound was identified as emodin.

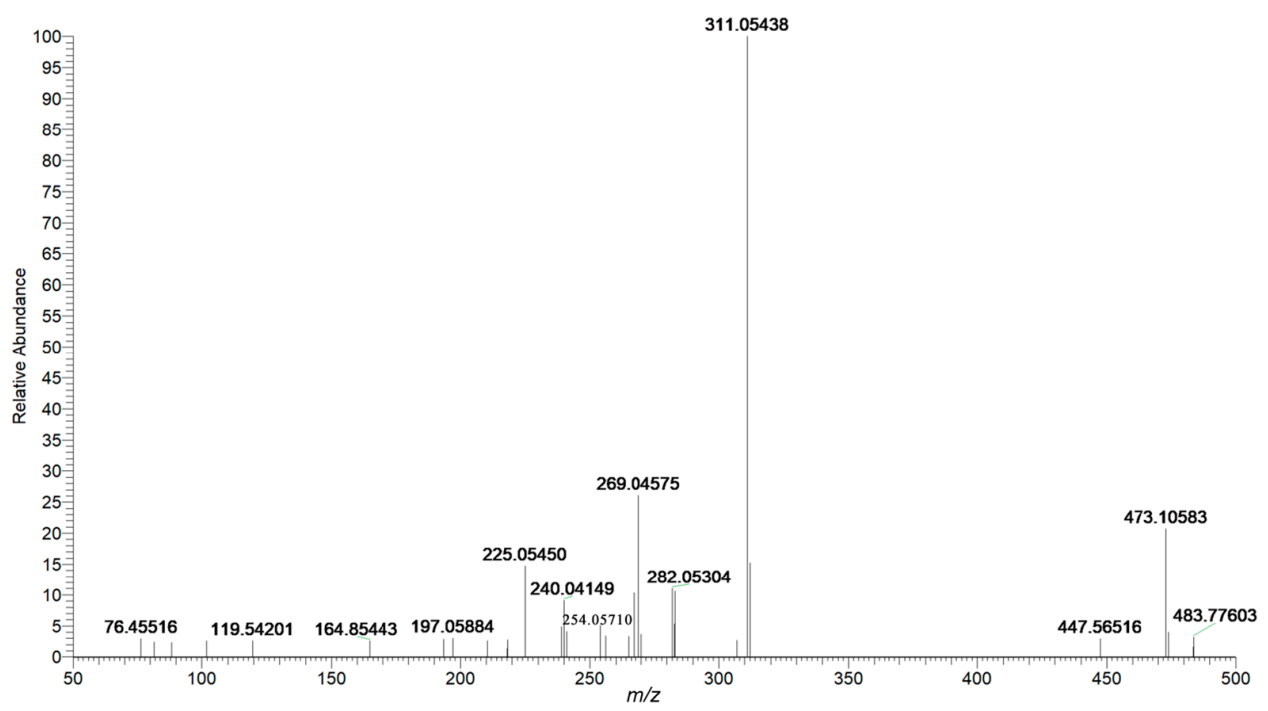

Figure 3. Mass spectrum of 2-acetylemodin-8-O- $\beta$-D-glucoside in negative mode.

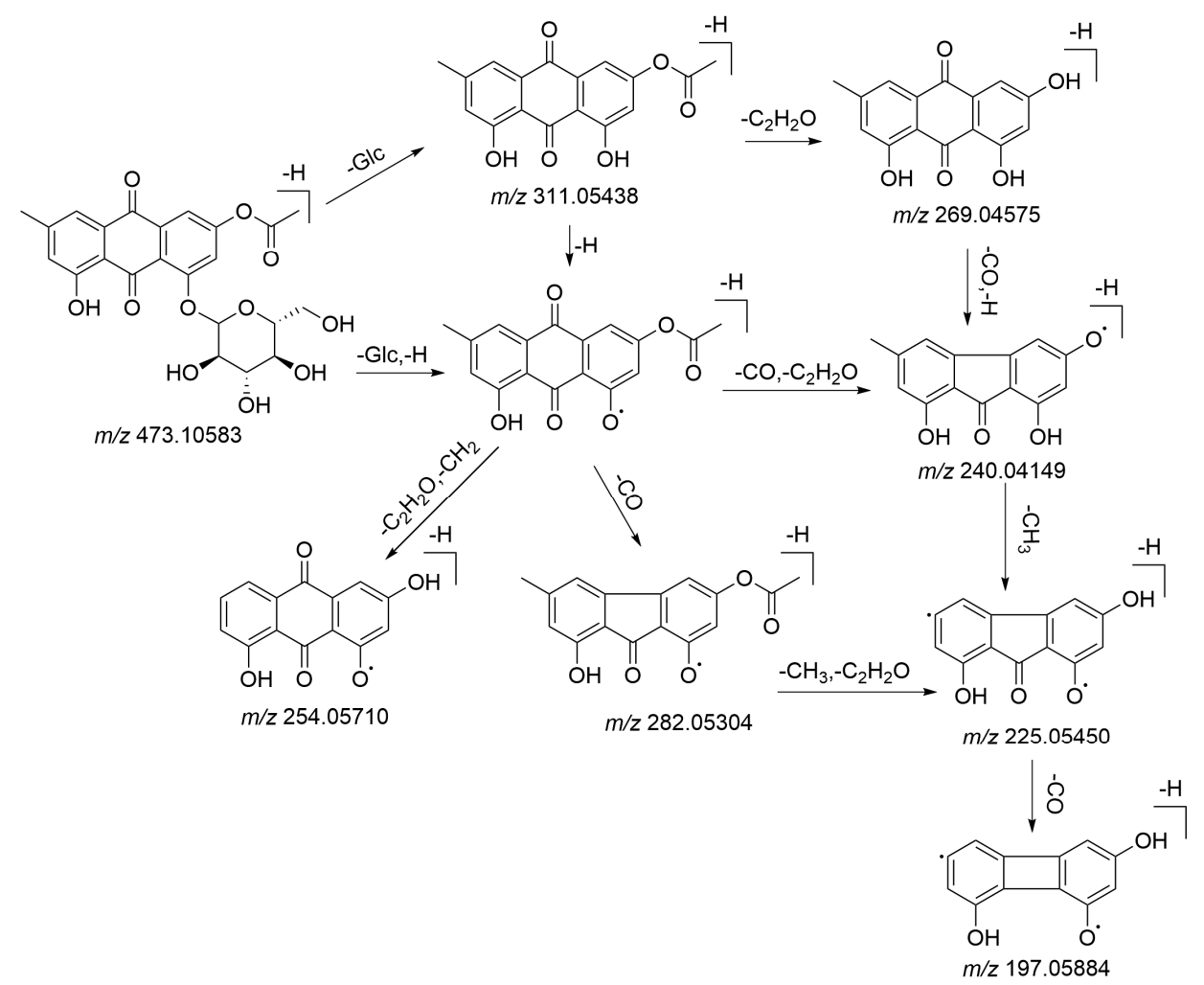

Figure 4. The proposed fragmentation pathways of 2 -acetylemodin-8- $O$ - $\beta$-D-glucoside. 


\subsubsection{Identification of Stilbenes and Derivatives}

Stilbenes are the main characteristic components in Polygoni Multiflori Radix Praeparata, showing great lipid-regulating and antioxidant activity [8]. Specifically, THSG as a unique active constituent plays a vital role in hepatoprotective effects, with various abilities as to the improvement of mitochondrial function and the clearance of intracellular reactive oxygen species $[32,33]$. On the other hand, some studies have reported that THSG was regarded as a contributor to liver injury associated with the transformation of trans-THSG to cis-THSG [34]. Stilbenes and its derivatives displayed characteristic fragment ions at $m / z 405$ and $m / z 243$ in negative ion mode. The other two prominent ions at $m / z 225$ and $m / z 215$ were obtained as loss $\mathrm{CO}$ and $\mathrm{H}_{2} \mathrm{O}$ in A-ring after rearrangement, respectively. In detail, peak 52 was found at $7.94 \mathrm{~min}$ and generated an $[\mathrm{M}-\mathrm{H}]^{-}$ion at $m / z$ 405.11670. The characteristic ion at $m / z 243.06503$ was produced by losing $\mathrm{C}_{6} \mathrm{H}_{10} \mathrm{O}_{5}$ from the precursor ion. Other characteristic ions at $\mathrm{m} / z 225.05450$ and 215.07039 were obtained by losing $\mathrm{H}_{2} \mathrm{O}$ and $\mathrm{CO}$ from $m / z 243.06503$, respectively. Compound 52 was identified as THSG by comparing the reference standard. Figure 5 shows the MS/MS mass spectrum of THSG. The details of proposed fragmentation pathways are depicted in Figure 6. Peaks 67 and 69 were observed at $9.57 \mathrm{~min}$ and $9.95 \mathrm{~min}$, respectively. Their molecular formulas were predicted as $\mathrm{C}_{27} \mathrm{H}_{26} \mathrm{O}_{13}$ within $5 \mathrm{ppm}$. They all produced fragment ions at $m / z 405.11,243.06$ and 225.05, which were indicated as [M-H-gal $]^{-},[\mathrm{M}-\mathrm{H}-\text { gal-glc }]^{-},\left[\mathrm{M}-\mathrm{H}-\right.$ gal-glc- $\mathrm{H}_{2} \mathrm{O}^{-}$, respectively. Although it was difficult to distinguish them by MS spectra, it was easier to identify them by comparing their retention time. According to the in-house database, the two compounds were Tetrahydroxy-stilbene-O-(galloyl)-glucoside and Piceatannol-3-O- $\beta$ D-(6"-O-galloyl)-glucoside. Based on the different positions of hydroxy in the benzene ring, the dehydration ability of them was different. Tetrahydroxystilbene-O-(galloyl)-Glucoside is more polar and can be more quickly eluted than Piceatannol-3-O- $\beta$-D-(6"-O-galloyl)glucoside on reserved phase column. Therefore, peak 67 was putatively identified as Tetrahydroxystilbene-O-(galloyl)-Glucoside, and peak 69 was Piceatannol-3-O- $\beta$-D-(6"$O$-galloyl)-glucoside. Peak 68 was found at $9.87 \mathrm{~min}$ and generated $[\mathrm{M}-\mathrm{H}]^{-}$ion at $\mathrm{m} / \mathrm{z}$ 447.12930. MS/MS fragment at $m / z 243.06511$ and 225.05455 corresponded to [M-Hglc-acetyl] ${ }^{-}$and $\left[\mathrm{M}-\mathrm{H}-\text { glc-acetyl- } \mathrm{H}_{2} \mathrm{O}\right]^{-}$. Compound 68 was putatively identified as 2,3,5,4'-tetrahydroxy-stilbene-2-O-(2"-O-acetyl)- $\beta$-D-glucoside.

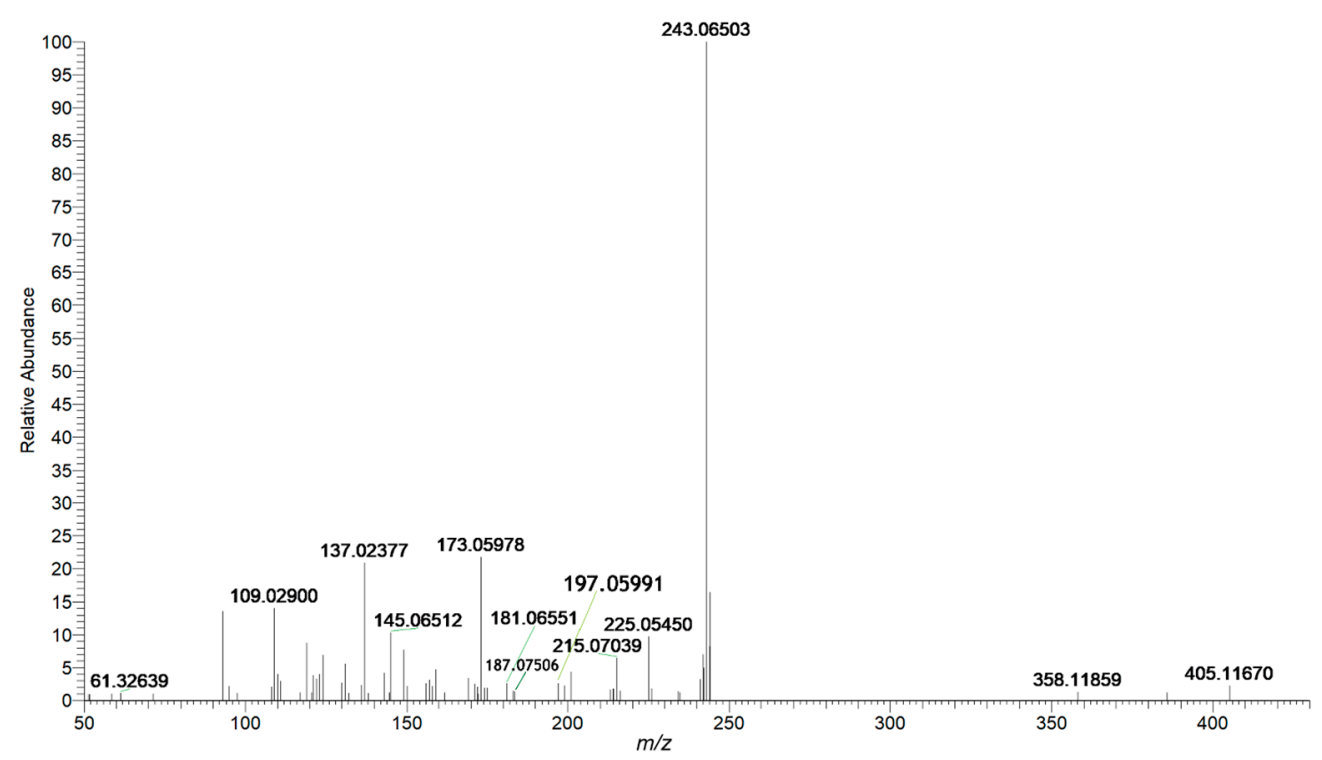

Figure 5. Mass spectrum of THSG in negative mode. 


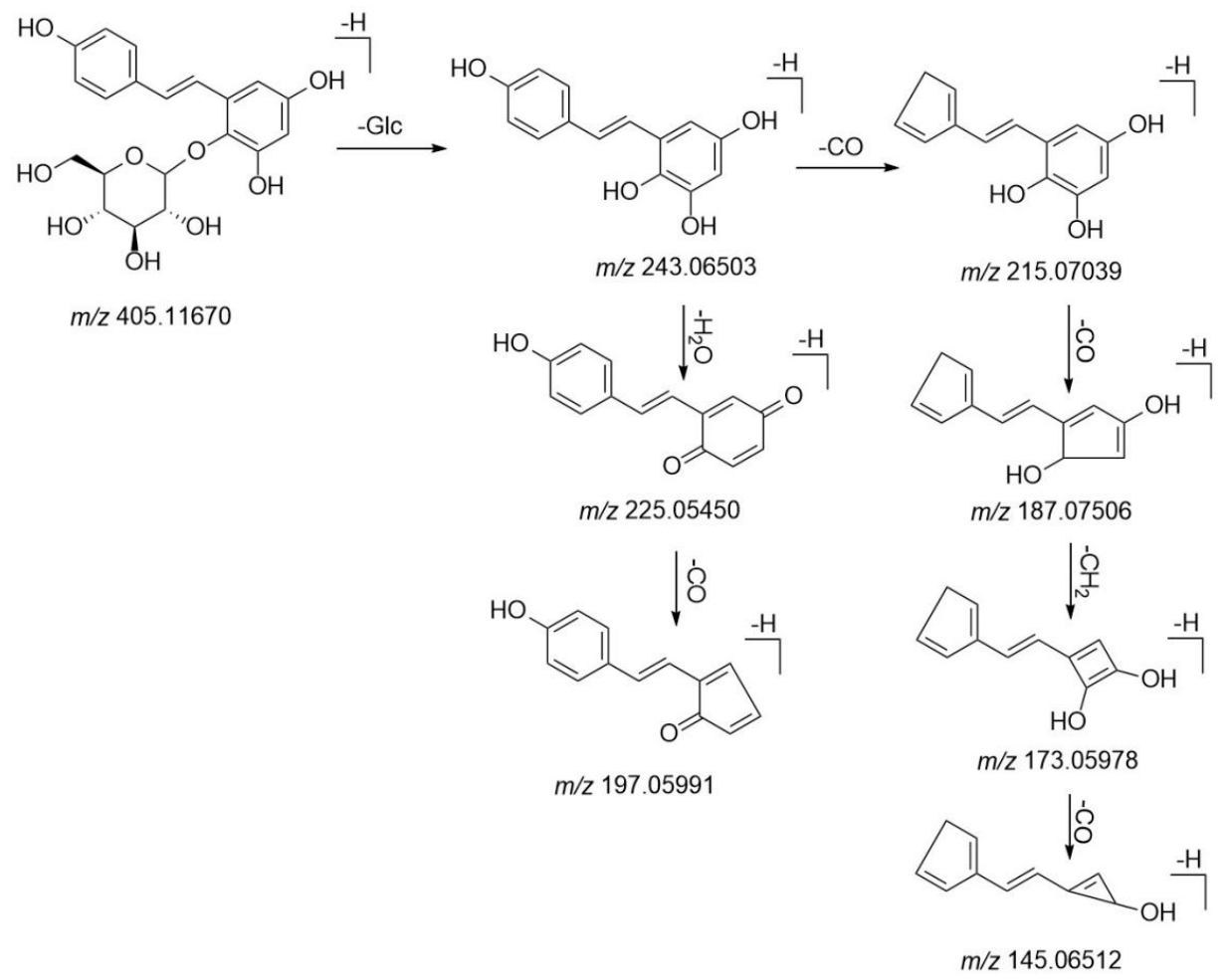

Figure 6. The proposed fragmentation pathways of THSG.

\subsubsection{Identification of Flavonoids and Derivatives}

As the main antioxidant in the root, flavonoids and their derivatives exhibit antioxidant and free radical scavenging activities [35]. In addition, flavonoids can protect against liver injury through the regulation of NF- $\mathrm{KB} / \mathrm{I \kappa B} \alpha$, p38 MAPK, and Bcl-2/Bax signaling [36]. There were 14 compounds tentatively identified as flavonoids and their derivatives. Catechin and epicatechin are isomers and they were used as examples to illustrate the characterization process of flavonoids, which can undergo an RDA reaction by cleavage of $\mathrm{C} 3-\mathrm{C} 4$ and $\mathrm{C} 2-\mathrm{C} 1$ bonds of the $\mathrm{C}$ ring rearranging, and produced the characteristic fragment ions of $m / z 151$ and $m / z$ 137. Figure 7 shows the MS/MS mass spectrum of epicatechin. The proposed fragmentation pathways are depicted in Figure 8. Peak 28 and Peak 31 generated an $[\mathrm{M}-\mathrm{H}]^{-}$ion at $m / z 289.07111$ and 289.07080, respectively. Their molecular formulas were all predicted as $\mathrm{C}_{15} \mathrm{H}_{14} \mathrm{O}_{6}$ within $5 \mathrm{ppm}$. The common characteristic ions were observed at $m / z 151.03,37.02,123.04$. The precursor ions undergo an RDA reaction to produce $m / z 151.03$ and 137.02 , corresponding to $\left[\mathrm{M}-\mathrm{H}-\mathrm{C}_{7} \mathrm{H}_{5} \mathrm{O}_{3}\right]^{-}$and $\left[\mathrm{M}-\mathrm{H}-\mathrm{C}_{8} \mathrm{H}_{7} \mathrm{O}_{3}\right]^{-}$, respectively. Characteristic ions at $\mathrm{m} / z 123.04$ were obtained by losing $\mathrm{CO}$ from $\mathrm{m} / \mathrm{z}$ 311.05438. Based on their different hydroxyl configuration at the $\mathrm{C} 3$ position and combined with the literature, catechin is more polar than epicatechin. Therefore, in this separation condition, the retention time of catechin is shorter. Peak 28 was putatively identified as catechin. Peak $\mathbf{3 1}$ was epicatechin. 


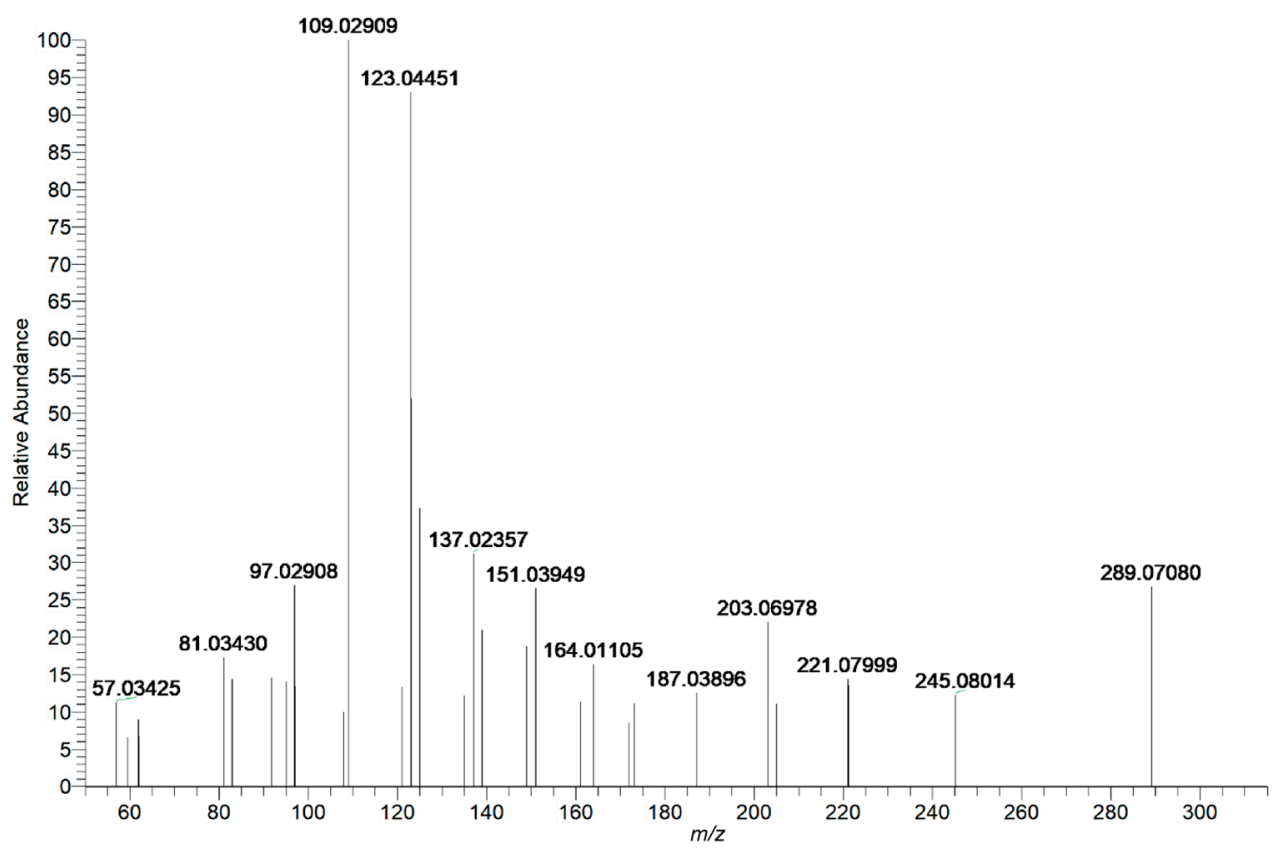

Figure 7. Mass spectrum of epicatechin in negative mode.<smiles>Oc1cc(O)c2c(c1)OC(c1ccc(O)c(O)c1)C(O)C2</smiles>

$\mathrm{m} / \mathrm{z} 289.07080$

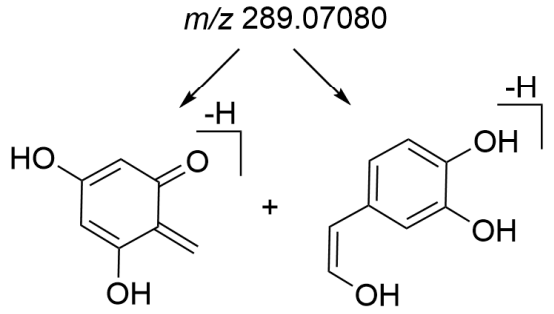
$\mathrm{m} / \mathrm{z} 137.02357$<smiles>[CH]C</smiles>
$\mathrm{HO}$<smiles>C=C1C(C)=CC=C1O</smiles>
$\mathrm{m} / \mathrm{z} 109.02909$

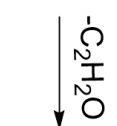<smiles>CC(=O)O</smiles>
$\mathrm{m} / \mathrm{z} 151.03949$<smiles>CCCCCCCCCCCCOc1ccccc1O</smiles>

$\mathrm{HO}$<smiles>Oc1cc(O)c2c(c1)OC(C1=CCC=C1)C(O)C2</smiles>

$\mathrm{m} / \mathrm{z} 245.08014$<smiles>OC1Cc2c#cccc2OC1c1ccc2ccccc2c1</smiles>

m/z 221.07999<smiles>C1=CC(c2ccccc2)Oc2ccccc21</smiles>

$\mathrm{m} / \mathrm{z} 203.06978$<smiles>Cc1ccc(C2C=Cc3ccccc32)cc1</smiles>

$\mathrm{m} / \mathrm{z} 187.03896$

Figure 8. The proposed fragmentation pathways of epicatechin. 


\subsubsection{Identification of Phenolic Acids and Derivatives}

Fifteen phenolic acids and their derivatives were tentatively identified in PMRP. It has been reported to exhibit hepatoprotective effects and good inhibitory activity towards $\alpha$ glucosidase[37]. Phenolic acids were structures containing one or more phenolic hydroxyl moieties. Therefore, the loss of $44 \mathrm{Da}(-\mathrm{COO})$ and $18 \mathrm{Da}\left(\mathrm{H}_{2} \mathrm{O}\right)$ could be considered as the characteristic fragment behavior of phenolic acids and its derivatives. Gallic acid was reported as a potentially xenobiotic component with anti-inflammatory activities and hepatotoxicity[38,39]. As shown in Figure 9, peak 9 with a retention time of 1.99 min generated an $[\mathrm{M}-\mathrm{H}]^{-}$ion with mass accuracy at $m / z$ 169.01358. The characteristic ion at $\mathrm{m} / z 125.02383$ was produced by losing $\mathrm{CO}_{2}$ from the precursor ion. The consecutive loss of $\mathrm{CO}_{2}$ and $\mathrm{H}_{2} \mathrm{O}$ leads to characteristic ions at $m / z 125.02383$ and 107.01351. Compound 9 was identified as gallic acid by comparing with the reference standard. The proposed fragmentation pathways of gallic acid are illustrated in Figure 10. Peak $\mathbf{1 7}$ was found at 3.34 min and generated an $[\mathrm{M}-\mathrm{H}]^{-}$ion at $m / z$ 315.06995. It produced fragment ion at $m / z$ 153.01865 by losing $162 \mathrm{Da}$ which be considered as a loss of $\mathrm{C}_{6} \mathrm{H}_{10} \mathrm{O}_{5}$ group, and ion at $\mathrm{m} / \mathrm{z}$ 109.02901 by losing a $\mathrm{CO}_{2}(44 \mathrm{Da})$. Therefore, compound 17 could be tentatively identified as protocatechuic acid-O-glucoside.

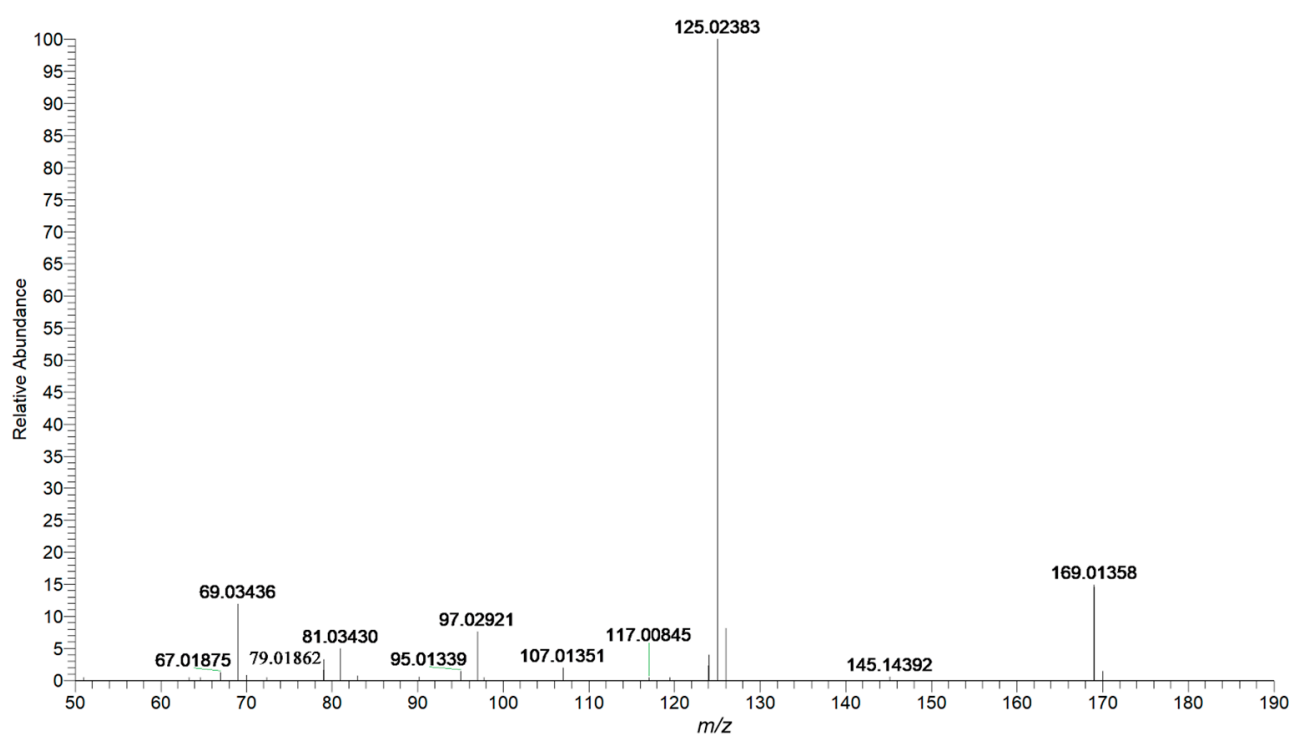

Figure 9. Mass spectrum of gallic acid in negative mode. 
<smiles>CC(C)C(=O)OCc1cc(O)c(O)c(O)c1</smiles>

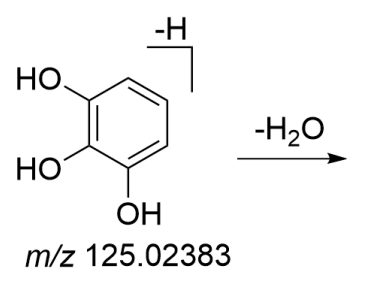<smiles>Oc1ccccc1O</smiles>

$m / z 169.01358$<smiles>OC1C=CC=C1</smiles><smiles>CC#CCC</smiles>

$m / z 69.03436$

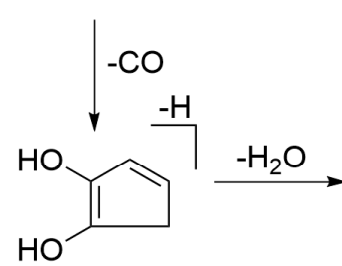<smiles>[13CH3]O[13CH3]</smiles>

$m / z 97.02921$

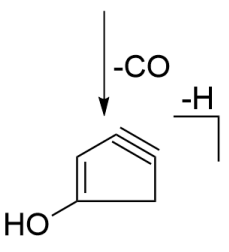

$m / z 79.01862$

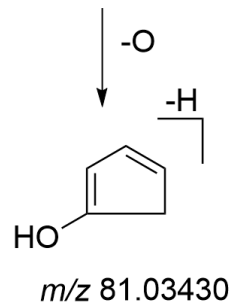

Figure 10. The proposed fragmentation pathways of gallic acid.

\subsubsection{Identification of Other Compounds}

Peak 13 generated an $[\mathrm{M}-\mathrm{H}]^{-}$ion at $m / z$ 125.02386, and the molecular formula was predicted as $\mathrm{C}_{6} \mathrm{H}_{6} \mathrm{O}_{3}$ within $5 \mathrm{ppm}$. Diagnostic ions at $m / z 97.02918$ and 81.03435 indicated the loss of $\mathrm{CO}$ and $\mathrm{CO}_{2}$ groups, respectively. Peak 13 was putatively identified as 5-Hydroxymethylfurfural, matched with the in-house database. Peak 22 was found at $4.35 \mathrm{~min}$ and produced an $[\mathrm{M}-\mathrm{H}]^{-}$ion at $\mathrm{m} / \mathrm{z} 189.05476$. The diagnostic fragment ions such as $m / z 174.03186$ and 161.06018 corresponded to $\left[\mathrm{M}-\mathrm{H}-\mathrm{CH}_{3}\right]^{-}$and $[\mathrm{M}-\mathrm{H}-\mathrm{CO}]^{-}$. Compound 22 was tentatively identified as Altechromone A. The $[\mathrm{M}-\mathrm{H}]^{-}$ion at $m / z$ 151.03902, which was found at $6.54 \mathrm{~min}$ in peak 36, indicated a molecular formula of $\mathrm{C}_{8} \mathrm{H}_{8} \mathrm{O}_{3}$ within $5 \mathrm{ppm}$. It produced characteristic fragments at $m / z 136.01607,123.04456$, and 107.04993 due to the elimination of $\left[\mathrm{M}-\mathrm{H}-\mathrm{CH}_{3}\right]^{-},[\mathrm{M}-\mathrm{H}-\mathrm{CO}]^{-}$and $\left[\mathrm{M}-\mathrm{H}-\mathrm{CO}_{2}\right]^{-}$, respectively. Finally, peak 36 was tentatively identified as Vanillin. Aside from the major compounds analyzed above, the remaining constituents were also identified by comparing them with the in-house database.

\subsubsection{Comparison of Chemical Constituents Between PMRPE and PMRPW}

Based on the identification strategy we have established, 101 components were identified in PMRPE and 91 components were identified in PMRPW. The results showed that there were 12 characteristic components in PMRPE, which were 2-vinyl-1H-indole-3-carboxylic acid, syringic acid, epicatechin-O-gallate, 2-methyl-5-carboxymethyl-7-hydroxychromone, epimedium, 2,3,5,4'-tetrahydroxystilbene-2-O-(2"'-O-acetyl)- $\beta$-D-glucoside, 2,3,5,4'-tetrahydroxystilbene-2$O$ - $\beta$-D-(2"-O-coumaroyl)-glucoside, tetrahydroxystilbene-O-(caffeoyl)-glucoside, torachrysone, emodin-O-glucoside-gallate, chrysophanol anthrone and digitolutein. Two characteristic constituents were identified in PMRPW, which were polygonumosides $\mathrm{C}$ and di-emodindi-glucoside.

Meanwhile, the peak area of potentially xenobiotic compounds reported in previous studies has been compared in PMRPW and PMRPE [1,40,41]. The representative chromatograms of potentially xenobiotic compounds are shown in Figure 11, and the specific parameters are listed in Table 2. 


\section{PMRPW}
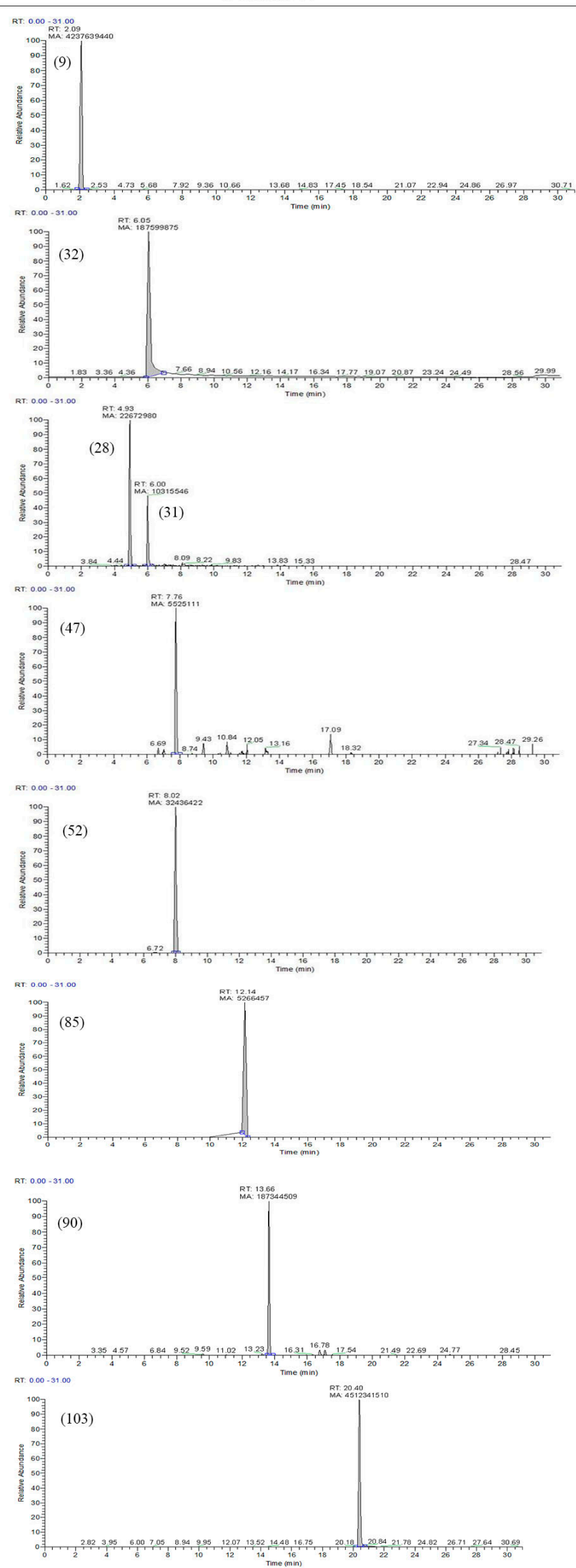

PMRPE
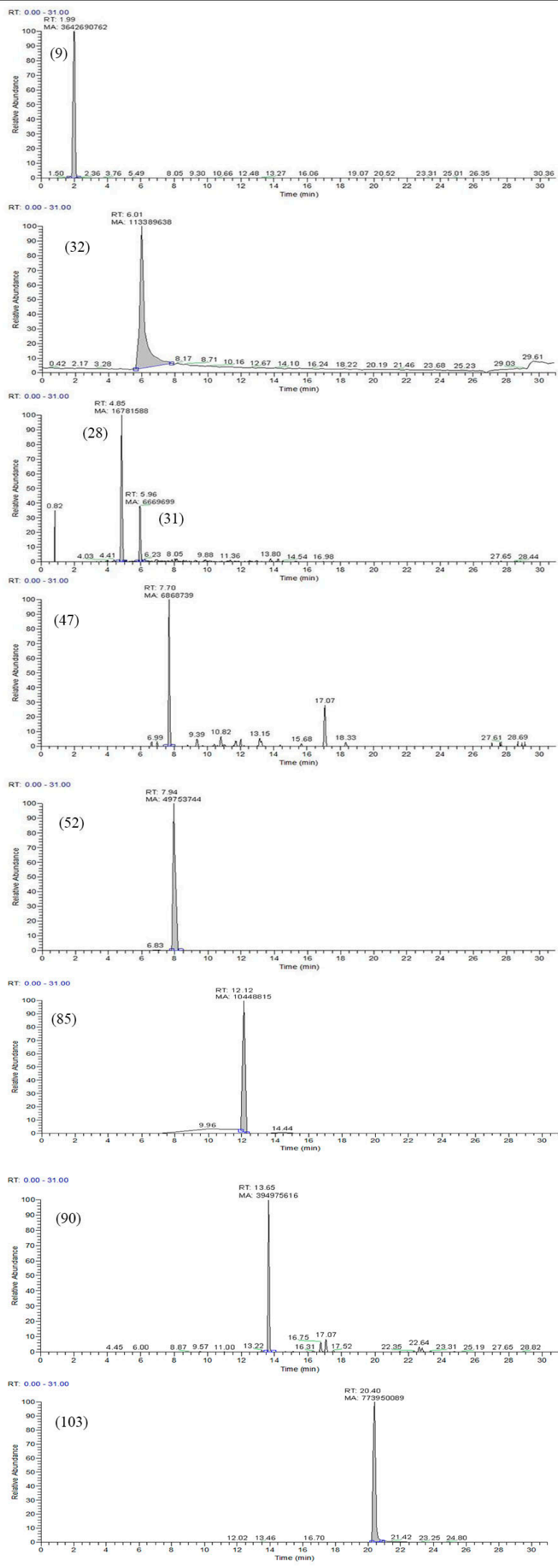

Figure 11. The chromatograms of potentially xenobiotic compounds in PMRPW and PMRPE. 
Table 2. The peak area of potentially xenobiotic compounds in PMRPW and PMRPE.

\begin{tabular}{|c|c|c|c|c|c|c|c|c|}
\hline \multirow{2}{*}{ Peak No. } & \multirow{2}{*}{ RT } & \multirow{2}{*}{$\begin{array}{l}\text { Compound } \\
\text { Name }\end{array}$} & \multirow{2}{*}{$\begin{array}{l}\text { Molecular } \\
\text { Formula }\end{array}$} & \multicolumn{2}{|c|}{ Area } & \multicolumn{2}{|c|}{ Percentage of the Area } & \multirow{2}{*}{ Mechanisms of Hepatotoxicity } \\
\hline & & & & PMRPW & PMRPE & PMRPW & PMRPE & \\
\hline 9 & 1.99 & Gallic acid & $\mathrm{C}_{7} \mathrm{H}_{6} \mathrm{O}_{5}$ & $4,237,639,440$ * & $3,642,690,762$ & $99.93 \%$ & $99.91 \%$ & CYP1A2 $\downarrow ;$ Caspase- $3 \uparrow$ \\
\hline 28 & 4.79 & Catechin & $\mathrm{C}_{15} \mathrm{H}_{14} \mathrm{O}_{6}$ & $22,672,980 *$ & $16,781,588$ & $95.54 \%$ & $96.05 \%$ & UGT1A6 $\downarrow ;$ UGT2B1 $\downarrow$ \\
\hline 31 & 5.93 & Epicatechin & $\mathrm{C}_{15} \mathrm{H}_{14} \mathrm{O}_{6}$ & $10,315,546$ * & $6,669,699$ & $92.05 \%$ & $90.44 \%$ & Caspase-3 $\uparrow ;$ UGT1A6 $\uparrow ;$ UGT2B1 $\downarrow$ \\
\hline 47 & 7.76 & Resveratrol & $\mathrm{C}_{14} \mathrm{H}_{12} \mathrm{O}_{3}$ & $5,525,111$ & $6,868,739 *$ & $76.05 \%$ & $74.84 \%$ & Cyp7a1 $\uparrow$ \\
\hline 52 & 7.94 & THSG & $\mathrm{C}_{20} \mathrm{H}_{22} \mathrm{O}_{9}$ & $32,436,422$ & $49,753,744$ * & $96.08 \%$ & $95.11 \%$ & $\begin{array}{l}\text { Cyp7a1 } \uparrow ; \text { Hmgcr } \uparrow \\
\text { CytP-450 } \downarrow ; \text { UDP } \downarrow\end{array}$ \\
\hline 85 & 12.12 & EG & $\mathrm{C}_{21} \mathrm{H}_{20} \mathrm{O}_{10}$ & $5,266,457$ & $10,448,815$ * & $97.11 \%$ & $96.42 \%$ & $\mathrm{UDP} \downarrow ; \mathrm{CYP} 2 \mathrm{~A} \downarrow ; \mathrm{UGT} 1 \mathrm{~A} 1 \downarrow ; \mathrm{CYP} 3 \mathrm{~A} 4 \downarrow$ \\
\hline 90 & 13.65 & Physcion & $\mathrm{C}_{16} \mathrm{H}_{12} \mathrm{O}_{5}$ & $187,344,509$ & $394,975,616^{*}$ & $94.04 \%$ & $95.77 \%$ & Cyp8b1 $\downarrow ;$ Cyp7a1 $\uparrow$ \\
\hline 103 & 20.37 & Emodin & $\mathrm{C}_{15} \mathrm{H}_{10} \mathrm{O}_{5}$ & $4,512,341,510$ & $773,950,089 *$ & $99.71 \%$ & $99.28 \%$ & $\begin{array}{l}\text { CytP-50 } \downarrow ; \text { Cyp8b1 } \downarrow \\
\text { Cyp7a1 } \uparrow ; \text { CYP3A4 } \downarrow\end{array}$ \\
\hline
\end{tabular}

*: means the peak area of this group is higher than the other group; " $\uparrow ":$ means promoting expression; " $\downarrow$ ": means inhibiting expression.

\section{Materials and Methods}

\subsection{Materials and Chemicals}

HPLC grade acetonitrile (ACN) and acetic acid were purchased from Sigma-Aldrich (St. Louis, MO, USA), and Dikma Technology Co., Ltd. (Beijing, China). Ethanol (industrial grade) was obtained from Shandong Yuwang Industry Co., Ltd. (Shandong, China). Purified water was obtained from the Hangzhou Wahaha Corporation (Hangzhou, China). PMRP was bought from GuoDa Pharmacy (Shenyang, Liaoning, China) and was identified as the processed root of Polygonum multiflorum Thunb. by professor Zhiguo Yu of the Shenyang Pharmaceutical University. The reference standards of emodin, physcion, and gallic acid were purchased from Chengdu MUST Bio-Technology Co., Ltd. (Sichuan, China). Chrysophanol, rhein and polydatin were obtained from the National Institute for the Control of Pharmaceutical and Biological Products (NICPBP, Beijing, China). Emodin-8$O$ - $\beta$-D-glucoside and 2,3,5,4-tetrahydroxystilbene-2-O- $\beta$-D-glucoside (THSG) were obtained from Sichuan Victory Bio-Technology Co., Ltd. (Sichuan, China). The purity of all the reference substances was higher than $98 \%$.

\subsection{Standard Solutions and Sample Preparation}

Standard solution: each reference standard was accurately weighed and dissolved in methanol as the stock solutions. Afterward, appropriate amounts of eight stock solutions were mixed and diluted into a suitable working solution with methanol before it was used.

Sample solution: $100 \mathrm{~g}$ of PMRP was soaked for $1 \mathrm{~h}$ with $1000 \mathrm{~mL} \mathrm{70 \%} \mathrm{aqueous}$ ethanol solution as soaking solvent and then refluxed for $2 \mathrm{~h}$. After being filtered with gauze, the residue was refluxed twice with another $800 \mathrm{~mL}$ of $70 \%$ aqueous ethanol solution for $1 \mathrm{~h}$. Finally, the filtrates were mixed and evaporated to $0.5 \mathrm{~g} / \mathrm{mL}$ as PMRPE in a rotary evaporator. The preparation method of PMRPW was the same as that of PMRPE, except that $70 \%$ aqueous ethanol solution was replaced with water. Appropriate PMRPE and PMRPW extract were diluted with $50 \%$ aqueous methanol solution to obtain $15 \mathrm{mg} / \mathrm{mL}$ solution, respectively (calculated as raw herbs). The solution was centrifuged at 13000 $\mathrm{rpm}$ for $10 \mathrm{~min}$ and filtered through a $0.22 \mu \mathrm{m}$ membrane. An aliquot of $5 \mu \mathrm{L}$ was injected for analysis.

\subsection{LC System and Mass Spectrometry}

LC analysis was performed on a Vanquish Flex UHPLC system (Thermo Fisher Scientific, Waltham, MA, USA) equipped with an HSS T3 column $(2.1 \mathrm{~mm} \times 100 \mathrm{~mm}$, $1.8 \mu \mathrm{m}$, Waters Corporation, Milford, UK). The sample chamber and column temperatures were maintained at $10{ }^{\circ} \mathrm{C}$ and $35^{\circ} \mathrm{C}$, respectively. The gradient elution with mobile phase acetonitrile (A) $-0.1 \%$ formic acid in water (B) was set as follows: $5-15 \%$ (A) from 0 to $4 \mathrm{~min}$; $15-50 \%$ (A) from 4 to $10 \mathrm{~min}$; 50-60\% (A) from 10 to $15 \mathrm{~min}$; 60-95\% (A) from 15 to $25 \mathrm{~min}$; $95 \%$ (A) from 25 to $28 \mathrm{~min}$; $95-5 \%$ (A) from 28 to $28.1 \mathrm{~min} ; 5 \%$ (A) from 28.1 to $31 \mathrm{~min}$. The flow rate was $0.3 \mathrm{~mL} / \mathrm{min}$, and the injection volume was $5 \mu \mathrm{L}$.

A Q-Exactive Orbitrap mass spectrometry instrument (Thermo Fisher Scientific, USA) was used to identify the constituents of PMRPE and PMRPW in negative modes. The 
mass spectrometer was set with the following parameters: spray voltage, $3.0 \mathrm{kV}$; capillary temperature, $350{ }^{\circ} \mathrm{C}$; auxiliary gas heater temperature, $350{ }^{\circ} \mathrm{C}$; sheath gas flow rate, $35 \mathrm{Arb}$; auxiliary gas flow rate, 10 Arb; S-lens RF level, $55 \mathrm{~V}$. The full scan and fragment spectra were collected at a resolution of 70,000 and 17,500, respectively. Full scan spectra were measured in a range from $m / z 80$ to 1200 . The automatic gain control (AGC) target and maximum injection time (IT) were $3 \times 10^{6}$ ions capacity and $50 \mathrm{~ms}$, respectively. For the $\mathrm{dd} \mathrm{MS}^{2}$ mode, the automatic gain control (AGC) target and maximum injection time (IT) were $1 \times 10^{5}$ ions capacity and $50 \mathrm{~ms}$. In each cycle, the top 10 precursor ions were chosen for fragmentation at collision energy (CE) of 20,40 and 60 V. Data were analyzed by using Xcalibur $^{\mathrm{TM}}$ version 2.2.1 and TraceFinder 4.1 version (Thermo Fisher Scientific, Waltham, MA, USA).

\section{Conclusions}

In this study, a rapid, sensitive, and specific analytical method was established using UHPLC-Q-Exactive Orbitrap-MS to identify the chemical constituents of PMRP. A total of 103 compounds, including 24 anthraquinones, 21 stilbenes, 15 phenolic acids, 14 flavones, and 29 other types were identified or tentatively characterized. There were 101 components in PMRPE and 91 components in PMRPW. Moreover, we have compared the peak areas of several significant components in PMRPW and PMRPE. The results showed that PMRPE has a higher content of anthraquinones and stilbenes than that of PMRPW. Previous studies have suggested that the hepatotoxicity of ethanol extract was stronger than that of water extract, indicating that anthraquinones and stilbenes might be the crucial xenobiotic components of liver injury induced by PMRP. Meanwhile, the specific toxicity compounds and mechanisms of hepatotoxicity also need further exploration. Considering the complex absorption and metabolism after oral administration, the characterization of PMRP's composition in vitro research was not enough. Therefore, the identification of chemical constituents in vivo and the verification of hepatotoxicity mechanisms of PMRP are still under investigation. In conclusion, the profiles of the constituents provide more information to understand PMRP from a chemical viewpoint and establish a substantial basis for further studies. The results also demonstrated that the novel method would be meaningful to the characterization of components in other botanical extracts.

Supplementary Materials: The following are available online, Figure S1: Chemical structures of compounds identified in PMRP.

Author Contributions: S.W. and Z.Y.; formal analysis, X.S.; investigation, S.W., S.A. and F.S.; software, S.W. and X.S.; writing — original draft, S.W. writing—-review and editing, S.W.; supervision, Y.Z. and Z.Y.; All authors have read and agreed to the published version of the manuscript.

Funding: This research did not receive any specific grant from funding agencies in the public, commercial, or not-for-profit sectors.

Institutional Review Board Statement: Not applicable.

Informed Consent Statement: Not applicable.

Conflicts of Interest: The authors declare no conflict of interest.

Sample Availability: Samples of the compounds are available from the authors.

\section{References}

1. Li, H.L.; Wang, X.B.; Liu, Y.; Pan, D.; Ye, W.; Nian, Y.; Xiang, L.C.; Cai, X.J.; Feng, Y.B. Hepatoprotection and hepatotoxicity of Heshouwu, a Chinese medicinal herb: Context of the paradoxical effect. Food Chem. Toxicol. 2017, 108, 407-418. [CrossRef] [PubMed]

2. Lin, L.F.; Ni, B.R.; Lin, H.M.; Zhang, M.; Li, X.; Yin, X.; Qu, C.; Ni, J. Traditional usages, botany, phytochemistry, pharmacology and toxicology of Polygonum multiflorum Thunb.: A review. J. Ethnopharmacol. 2015, 159, 158-183. [CrossRef]

3. Chen, Q.; Zhang, S.Z.; Ying, H.Z.; Dai, X.Y.; Li, X.-x.; Yu, C.-h.; Ye, H.-c. Chemical characterization and immunostimulatory effects of a polysaccharide from Polygoni Multiflori Radix Praeparata in cyclophosphamide-induced anemic mice. Carbohydr. Polym. 2012, 88, 1476-1482. [CrossRef] 
4. Thiruvengadam, M.; Praveen, N.; Kim, E.-H.; Kim, S.-H.; Chung, I.-M. Production of anthraquinones, phenolic compounds and biological activities from hairy root cultures of Polygonum multiflorum Thunb. Protoplasma 2014, 251, 555-566. [CrossRef] [PubMed]

5. Chen, L.L.; Huang, X.J.; Li, M.M.; Ou, G.M.; Zhao, B.-X.; Chen, M.-F.; Zhang, Q.-W.; Wang, Y.; Ye, W.-C. Polygonflavanol A, a novel flavonostilbene glycoside from the roots of Polygonum multiflorum. Phytochem. Lett. 2012, 5, 756-760. [CrossRef]

6. Yang, J.B.; Li, L.; Zhong, D.; Yu, W.; Xing-Chao, G.; Bo, L.; Shuang-Cheng, M.; Ai-Guo, W.; Ya-Lun, S. Polygonumnolides C1-C4; minor dianthrone glycosides from the roots of Polygonum multiflorum Thunb. J. Asian Nat. Prod. Res. 2016, 18, 813-822. [CrossRef] [PubMed]

7. Lv, L.S.; Tang, J. Structure-activity relationship of stilbene glycoside from Polygonum multiflorum Thunb. and resveratrol. Food Mach. 2009, 25, 57-58.

8. Lv, L.-S.; Gu, X.H.; Ho, C.T.; Tang, J. Stilbene glycosides from the roots of Polygonum multiflorum thunb and their in vitro antioxidant activities. J. Food Lipids 2006, 13, 131-144. [CrossRef]

9. Park, S.J.; Jin, M.L.; An, H.K.; Kim, K.-S.; Ko, M.J.; Kim, C.M.; Choi, Y.W.; Lee, Y.-C. Emodin induces neurite outgrowth through PI3K/Akt/GSK-3ß-mediated signaling pathways in Neuro2a cells. Neurosci. Lett. 2015, 588, 101-107. [CrossRef]

10. Choi, S.G.; Kim, J.; Sung, N.D.; Son, K.-H.; Cheon, H.-G.; Kim, K.-R.; Kwon, B.-M. Anthraquinones, Cdc25B phosphatase inhibitors, isolated from the roots of Polygonum multiflorum Thunb. Nat. Prod. Res. 2007, 21, 487-493. [CrossRef] [PubMed]

11. Wang, H.Y.; Song, L.X.; Feng, S.B.; Liu, Y.; Zuo, G.; Lai, F.; He, G.; Chen, M.; Huang, D. Characterization of Proanthocyanidins in Stems of Polygonum multiflorum Thunb as Strong Starch Hydrolase Inhibitors. Molecules 2013, 18, 2255-2265. [CrossRef]

12. Lei, X.; Chen, J.; Ren, J.T.; Li, Y.; Jingbo, Z.; Wei, M.; Li, Z.; Wenke, Z.; Guihua, T.; Hongcai, S. Liver Damage Associated with Polygonum multiflorum Thunb.: A Systematic Review of Case Reports and Case Series. Evid. Based Complement. Altern. Med. Ecam 2015, 2015, 459749. [CrossRef] [PubMed]

13. Cárdenas, A.; Restrepo, J.C.; Sierra, F.; Correa, G. Acute Hepatitis Due to Shen-Min: A Herbal Product Derived from Polygonum multiflorum. J. Clin. Gastroenterol. 2006, 40, 629-632. [CrossRef] [PubMed]

14. Victor, J.; Navarro, M.D.; John, R.; Senior, M.D. Drug-Related Hepatotoxicity. N. Engl. J. Med. 2006, 354, 731-739.

15. Teschke, R.; Wolff, A.; Frenzel, C.; Schulze, J. Review article: Herbal hepatotoxicity an update on traditional Chinese medicine preparations. Aliment. Pharmacol. Ther. 2014, 40, 32-50. [CrossRef] [PubMed]

16. Bai, J.R.; Zhang, Y.S.; Tang, C.; Hou, Y.; Xiaopeng, A.; Xiaorui, C.; Yi, Z.; Xiaobo, W.; Xianli, M. Gallic acid: Pharmacological activities and molecular mechanisms involved in inflammation-related diseases. Biomed. Pharmacother. 2021, $133,110985$. [CrossRef] [PubMed]

17. Berman, A.Y.; Motechin, R.A.; Wiesenfeld, M.Y.; Holz, M.K. The therapeutic potential of resveratrol: A review of clinical trials. NPJ Precis. Oncol. 2017, 1, 2783-2840. [CrossRef] [PubMed]

18. Vanderperren, B.; Rizzo, M.; Angenot, L.; Haufroid, V.; Jadoul, M.; Hantson, P. Acute Liver Failure with Renal Impairment Related to the Abuse of Senna Anthraquinone Glycosides. Ann. Pharmacother. 2005, 39, 1353-1357. [CrossRef] [PubMed]

19. Wang, J.B.; Zhao, Y.L.; Xiao, X.H.; Li, H.; Zhao, H.; Zhang, P.; Jin, C. Assessment of the renal protection and hepatotoxicity of rhubarb extract in rats. J. Ethnopharmacol. 2009, 124, 18-25. [CrossRef]

20. Li, C.Y.; Niu, M.; Bai, Z.F.; Zhang, C.E.; Zhao, Y.L.; Li, R.; Tu, C.; Li, H.; Jing, J.; Meng, Y.; et al. Screening for main components associated with the idiosyncratic hepatotoxicity of a tonic herb, Polygonum multiflorum. Front. Med. 2017, 11, 253-265. [CrossRef] [PubMed]

21. Zhang, L.; Liu, X.; Tu, C.; Li, C.; Song, D.; Zhu, J.; Zhou, Y.; Wang, X.; Li, R.; Xiao, X.; et al. Components synergy between stilbenes and emodin derivatives contributes to hepatotoxicity induced by Polygonum multiflorum. Xenobiotica 2020, 50, 515-525. [CrossRef] [PubMed]

22. Yu, J.; Xie, J.; Mao, X.J.; Wang, M.J.; Li, N.; Wang, J.; Zhaori, G.-t.; Zhao, R.-h. Hepatoxicity of major constituents and extractions of Radix Polygoni Multiflori and Radix Polygoni Multiflori Praeparata. J. Ethnopharmacol. 2011, 137, 1291-1299. [CrossRef]

23. Li, C.L.; Ma, J.; Zheng, L.; Li, H.; Li, P. Determination of emodin in L-02 cells and cell culture media with liquid chromatographymass spectrometry: Application to a cellular toxicokinetic study. J. Pharm. Biomed. Anal. 2012, 71, 71-78. [CrossRef] [PubMed]

24. Liu, Y.; Wang, Q.; Yang, J.B. Polygonum multiflorum Thunb.: A Review on Chemical Analysis, Processing Mechanism, Quality Evaluation, and Hepatotoxicity. Front. Pharmacol. 2018, 9, 364. [CrossRef] [PubMed]

25. Wu, X.Q.; Chen, X.Z.; Huang, Q.C.; Fang, D.M.; Li, G.; Zhang, G. Toxicity of raw and processed roots of Polygonum multiflorum. Fitoterapia 2012, 83, 469-475. [CrossRef] [PubMed]

26. Cui, Y.; Chen, L.J.; Huang, T.; Ying, J.; Li, J. The pharmacology, toxicology and therapeutic potential of anthraquinone derivative emodin. Chin. J. Nat. Med. 2020, 18, 425-435. [CrossRef]

27. Lee, B.H.; Huang, Y.Y.; Duh, P.D.; Wu, S.C. Hepatoprotection of emodin and Polygonum multiflorum against CCl4-induced liver injury. Pharm. Biol. 2012, 50, 351-359. [CrossRef] [PubMed]

28. Xu, X.C.; Lin, S.Z. Chinese herb Radix Polygoni Multiflori as a therapeutic drug for liver cirrhosis in mice. J. Ethnopharmacol. 2007, $114,199-206$.

29. Xu, X.C.; Lin, S.Z. Experimental advance of applying emodin for prevention and treatment of liver diseases. Chin. J. Integr. Tradit. West. Med. 2008, 28, 91-93.

30. He, Y.R.; Song, M.Z.; Wang, W.G.; Lin, P.; Li, Y.; Gu, W.; Yu, J.; Zhao, R. Chronic toxicity of both raw and processed Polygoni Multiflori Radix on rats. J. Chin. Pharm. Sci. 2016, 25, 46-56. 
31. Lin, L.F.; Lin, H.M.; Zhang, M.; Ni, B.R.; Yin, X.B.; Qu, C.H.; Jian, N. A novel method to analyze hepatotoxic components in Polygonum multiflorum using ultra-performance liquid chromatography-quadrupole time-of-flight mass spectrometry. J. Hazard. Mater. 2015, 299. [CrossRef] [PubMed]

32. Büchter, C.; Zhao, L.; Havermann, S.; Honnen, S.; Fritz, G.; Proksch, P.; Wätje, W. TSG (2,3,5,4'-Tetrahydroxystilbene-2-O- $\beta$-Dglucoside) from the Chinese Herb Polygonum multiflorum Increases Life Span and Stress Resistance of Caenorhabditis elegans. Perspect. Vasc. Surg. Endovasc. Ther. 2015, 2015.

33. Qiao, S.Y.; Zhang, C.P.; Gao, X.Q.; Qiao, X.H.; Huang, L.Z. Effects of 2,3,5,4'-tetrahydroxystilbene-2-O- $\beta$-D-glucoside on the expression of NF- $\mathrm{kB} / \mathrm{I} \kappa \mathrm{B}$ and apoptosis of HUVECs induced by $\mathrm{H}_{2} \mathrm{O}_{2}$. Chin. Pharmacol. Bull. 2011, 27, $1353-1357$.

34. Hu, X.; Gao, J.; Li, Y.; Wang, J.; Wang, L. Effects of Stilbene Glucoside From Polygonum Multiflorum Thunb. on Hepatic Enzymes and Serum Albumin of Rats. Liaoning J. Tradit. Chin. Med. 2011, 38, 988-990.

35. Li, J.K.; Jiang, Z.Y.; Li, R.; Tan, J. Investigation of antioxidant activities and free radical scavenging of flavonoids in leaves of polygonum multiflorum thumb. China Food Addit. 2012, 69-74.

36. Wang, R.; Zhang, H.; Wang, Y.; Song, F.; Yuan, Y. Inhibitory effects of quercetin on the progression of liver fibrosis through the regulation of NF-kB/IkB $\alpha$, p38 MAPK, and Bcl-2/Bax signaling. Int. Immunopharmacol. 2017, 47, 126-133. [CrossRef] [PubMed]

37. Yang, J.B.; Tian, J.Y.; Dai, Z.; Ye, F.; Ma, S.; Wang, A. a -Glucosidase inhibitors extracted from the roots of Polygonum multiflorum Thunb. Fitoterapia 2017, 117, 65-70. [CrossRef] [PubMed]

38. Quan, Z.Y.; Li, D.K.; Li, Y.Q.; Wang, C.Y.; Zhou, M.; Hu, Y.H.; Sun, Z.X. CYP1A2 Depletion by shRNA Enhances Hepatotoxicity of Extract of Polygoni Multiflori Radix and Related Monomer Compositions. Chin. J. Pharmacovigil. 2021, 18, $213-219$.

39. Yessica, Z.C.; Andonegui-Elguera, M.A.; Ariadna, A.J.; Edson, A.S.; Karina, M.F.; Erika, R.P.; Cristina, V.M.; Clemente, I.; Valentín, M.L.; Marwin, G.; et al. The enzymatic poly(gallic acid) reduces pro-inflammatory cytokines in vitro, a potential application in inflammatory diseases. Inflammation 2020, 44, 174-185.

40. Xue, X.Y.; Quan, Y.Y.; Gong, L.H.; Gong, X.H.; Li, Y. A review of the processed Polygonum multiflorum (Thunb.) for hepatoprotection: Clinical use, pharmacology and toxicology. J. Ethnopharmacol. 2020, 261. [CrossRef]

41. Ruan, L.; Li, M.; Xing, Y.; Hong, W.; Chen, C.; Chen, J.; Xu, H.; Zhao, W.-L.; Wang, J.-S. Hepatotoxicity and hepatoprotection of Polygonum multiflorum Thund. as two sides of the same biological coin. J. Ethnopharmacol. 2019, 230, 81-94. [CrossRef] [PubMed] 